\title{
Antiwindup for Stable Linear Systems With Input Saturation: An LMI-Based Synthesis
}

\author{
Gene Grimm, Member, IEEE, Jay Hatfield, Ian Postlethwaite, Fellow, IEEE, Andrew R. Teel, Fellow, IEEE, \\ Matthew C. Turner, Member, IEEE, and Luca Zaccarian
}

\begin{abstract}
This paper considers closed-loop quadratic stability and $\mathcal{L}_{2}$ performance properties of linear control systems subject to input saturation. More specifically, these properties are examined within the context of the popular linear antiwindup augmentation paradigm. Linear antiwindup augmentation refers to designing a linear filter to augment a linear control system subject to a local specification, called the "unconstrained closed-loop behavior." Building on known results on $\mathcal{H}_{\infty}$ and LPV synthesis, the fixed order linear antiwindup synthesis feasibility problem is cast as a nonconvex matrix optimization problem, which has an attractive system theoretic interpretation: the lower bound on the achievable $\mathcal{L}_{2}$ performance is the maximum of the open and unconstrained closed-loop $\mathcal{L}_{2}$ gains. In the special cases of zero-order (static) and plant-order antiwindup compensation, the feasibility conditions become (convex) linear matrix inequalities. It is shown that, if (and only if) the plant is asymptotically stable, plant-order linear antiwindup compensation is always feasible for large enough $\mathcal{L}_{2}$ gain and that static antiwindup compensation is feasible provided a quasi-common Lyapunov function, between the open-loop and unconstrained closed-loop, exists. Using the solutions to the matrix feasibility problems, the synthesis of the antiwindup augmentation achieving the desired level of $\mathcal{L}_{2}$ performance is then accomplished by solving an additional LMI.
\end{abstract}

Index Terms-Antiwindup analysis, antiwindup synthesis, control systems, cost optimal control, finite $\mathcal{L}_{2}$ gain, linear matrix inequalities (LMIs), linear parameter varying (LPV).

\section{INTRODUCTION}

$\mathbf{P}$ ERHAPS the first problem in nonlinear control is to design high performance feedback algorithms for linear systems with input saturation. This task is theoretically challenging and,

Manuscript received July 31, 20001; revised October 11, 2002, January 20, 2003, and April 1, 2003. Recommended by Associate Editor V. Balakrishnan. The work of G. Grimm and A. R. Teel was supported in part by the Air Force Office of Scientific Research under Grant F49620-00-1-0106 and by the National Science Foundation under Grant ECS 9988813. The work of I. Postlethwaite and M. Turner was supported in part by the UK Engineering and Physical Sciences Research Council. The work of L. Zaccarian was supported in part by MIUR through Project MISTRAL and ASI under Grant I/R/152/00.

G. Grimm is with Raytheon Company, Space and Airborne Systems, El Segundo, CA 90245 USA.

J. Hatfield is with General Dynamics Land Systems, Goleta, CA 93117 USA (e-mail: jay.hatfield@gm.com)

I. Postlethwaite is with the Department of Engineering, the University of Leicester, Leicester LE1 7RH, U.K. (e-mail: ixp@le.ac.uk).

A. R. Teel is with the Electrical and Computer Engineering Department, University of California, Santa Barbara 93106-9560 USA (e-mail teel@ece.ucsb.edu).

M. C. Turner is with the Control and Instrumentation Research Group, the Department of Engineering, the University of Leicester, Leicester LE1 7RH, U.K. (e-mail: mct6@sun.engg.le.ac.uk).

L. Zaccarian is with DISP, University of Roma, Tor Vergata, 00133 Roma, Italy (e-mail: zack@disp.uniroma2.it).

Digital Object Identifier 10.1109/TAC.2003.816965 since actuator saturation is ubiquitous, it is critical for practical applications. Over the last decade considerable attention has been given to controlling linear systems with input saturation and significant progress has been reported in the literature.

The control objective for linear systems with input saturation becomes even more difficult to obtain when the behavior of the feedback algorithm must match a given behavior in the absence of input saturation. For example, the controller may need to be a particular PID controller for initial conditions and disturbances that do not trigger input saturation. A local requirement like this can arise for many reasons. In flight control, handling qualities specifications dictate local controller attributes. In vibration attenuation problems, frequency domain specifications constrain the local design. In general, it is common to encounter control problems where many years of experience have gone into the development of a small signal controller and an augmentation of that controller is desired to handle the effects of input saturation that appear occasionally. Augmentation is necessary when the predetermined controller is ill suited for the input saturation nonlinearity. Among early control algorithms, those that were most seriously affected by input saturation were those that contained integral action, e.g., PI or PID controllers. It was observed that, due to input saturation, the state of the integrator would "wind up" to excessively large values, leading to sluggish performance of the closed-loop control system [18]. It is for this reason that the phrase "antiwindup augmentation" is used to describe the problem of synthesizing controllers, subject to a local specification (called the unconstrained controller), for linear systems with input saturation.

As first noted in [5], the most typical embodiment of antiwindup augmentation has the form shown in Fig. 1, where $\mathcal{P}$ represents the linear plant and $\mathcal{C}$ represents the local controller specification. Due to the complexity of the antiwindup problem, where strict requirements for the small-signal behavior of the augmented system are combined with global (large-signal) stability, early antiwindup schemes were mostly heuristics and lacked mathematical rigor. (see, e.g., [11] and [2] for surveys of these early schemes). Only in the last decade has the problem been addressed in a more formal way with stability guarantees and clear performance specifications.

In [7], the antiwindup compensator synthesis problem was approached in a framework relying on $\mathcal{H}_{\infty}$ optimal control. The main thrust of this method was to interpret the performance of the resulting antiwindup compensator during saturation as an $\mathcal{L}_{2}$ gain minimization problem. The importance and practicality of the $\mathcal{L}_{2}$ norm was also recognized in [20], where stable plants were considered and a possible optimization procedure was suggested in terms of the $\mathcal{H}_{\infty}$ norms of certain transfer functions. In 


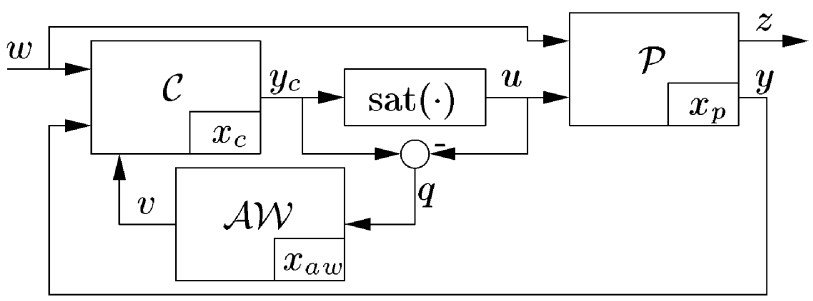

Fig. 1. Antiwindup augmentation scheme.

the work of [30], the induced $\mathcal{L}_{2}$ norm was linked directly with the behavior of the closed-loop system during saturation. Furthermore, various stability and performance tests for the closed loop system could be formulated as convex feasibility problems, for which efficient solvers are now available. In [29], a formal definition of the antiwindup problem was given. An important aspect of this definition was that recovery of linear performance (a concept also discussed in [6] and the references therein) was stated in terms of nonlinear $\mathcal{L}_{2}$ gains involving the unconstrained and the actual response of the system.

In recent years, several control applications started employing linear matrix inequalities (LMIs) [4] as a tool to exploit the (sometimes not evident) convexity of certain optimization problems in order to compute global optima in an extremely simplified way. Although many valid antiwindup constructions have been proposed, especially in the last decade that do not rely on LMIs (see, e.g., [10], [20], [27], [24], [15], and [13]), we will only focus here on LMI-based antiwindup designs.

While the control problem suggested by Fig. 1 is nonlinear, one way to tackle it is to treat it as a linear parameter varying (LPV) problem, where sat $\left(y_{c}\right)$ is replaced by $\Theta(t) y_{c}$ and $\Theta(\cdot)$ is a measurable, matrix-valued function taking values in a set consistent with reproducing the saturation nonlinearity. Within this approach, special care has to be taken in assuring the well-posedness of the interconnection around the nonlinearity. This is not an issue in the general LPV framework because $\Theta(t)$ is only a function of time. However, in the control problem in Fig. $1, \Theta(t)$ is actually better written as $\Theta\left(y_{c}(t)\right)$, and might result undefined if the system's response $y_{c}(t)$ is not well defined. We address and solve this well-posedness problem, in this paper, by means of a global nonsmooth inverse function theorem. The great advantage provided by the LPV framework is that quadratic stability and performance by means of fixed order antiwindup augmentation can be addressed using the LMI-based LPV synthesis ideas in [1] and [3] which derive from a combination of [23] and [8] (see also [12]). These synthesis ideas were applied to the control of linear systems with input saturation in [26] and [32], but not to what we have called the antiwindup augmentation problem since the control is not designed to match a given local controller.

The goal of this paper is to construct fixed-order dynamic antiwindup compensators which guarantee a given level of performance using suitable finite $\mathcal{L}_{2}$ gains of the augmented system as the performance objective (this was also considered in [22]). The basis for the study is the LMI-based $\mathcal{H}_{\infty}$ controller characterization of [8] and [12], where both full and reduced order controllers meeting an $\mathcal{H}_{\infty}$ norm-bound are described in terms of a nonconvex feasibility problem, which reduce to a convex feasibility problem when a certain rank constraint becomes inactive. When viewed in this LMI-based framework, the antiwindup augmentation design with $\mathcal{L}_{2}$ performance objective leads to nice system theoretic interpretations: a lower bound on the $\mathcal{L}_{2}$ gain achievable by the augmented system is the maximum of the $\mathcal{L}_{2}$ gains of the open-loop plant (with zero control input) and that of the unconstrained closed-loop system. Moreover, when the antiwindup compensator order is zero (static) or equal to the order of the plant (plant-order), the nonconvex matrix constraints can be reformulated in terms of (convex) LMI constraints that can be easily solved, optimizing globally the performance and providing simple and effective constructions for the antiwindup augmentation. Finally, by way of these new tools, plant-order augmentation can be shown to be always feasible (for large enough $\mathcal{L}_{2}$ gain), while static augmentation is feasible if and only if there exists a quasi-common quadratic Lyapunov function between the open-loop plant and the unconstrained closed-loop system. Moreover, asymptotic stability of the plant is shown to be a necessary condition for the global $\mathcal{L}_{2}$ performance requirement of this paper to be attainable.

LMI tools have been brought to bear on the antiwindup framework in very recent years. One of the earliest papers where LMIs and antiwindup were combined is [19] where stability and $\mathcal{L}_{2}$ performance analysis of closed-loop systems with static antiwindup compensation is formulated as an LMI problem amounting to the determination of a "simultaneous quadratic Lyapunov function." Moreover, [19] formulates the associated synthesis problem in terms of bilinear matrix inequalities. In [17], the stability analysis of more general antiwindup closed-loop systems arising from known antiwindup constructions were formulated in terms of LMIs and a first attempt to transform these LMI stability analysis tools into controller synthesis tools was made by the same authors in [16], where the modified mixed $\mathcal{H}_{2} / \mathcal{H}_{\infty}$ control problem was brought to bear in the static and dynamic antiwindup synthesis problem, noting that it was associated with nonlinear matrix inequalities. Only recently, a complete LMI formulation of the static antiwindup design problem, (namely, the case where the system $\mathcal{A W}$ in Fig. 1 is static, i.e., it has no dynamic state) was given in [22]. The result stops short of a system theoretic interpretation of the feasibility conditions for static antiwindup.

The main drawback of the static construction in [22] is that in several situations the LMI constraints are unfeasible. To address this problem, the same authors proposed an alternative static antiwindup design in [21], based on the approximate solution of nonlinear matrix inequalities, to relax the quadratic stability requirement to piecewise quadratic stability.

The rest of the paper is organized as follows. Section II gives a precise statement of the problem including a Lyapunov-based formulation of stability and performance. Section III gives the main results of this paper. In Section III-B, necessary and sufficient conditions for the existence of an antiwindup compensator guaranteeing stability and a given level of performance is given. Interesting connections between the existence of a suitable antiwindup compensator and properties of the open-loop plant and of the unconstrained closed-loop system are established based on this conditions. Furthermore, it is shown how, for some special values of the antiwindup compensator order, these conditions can be easily checked solving 
LMIs based on the unconstrained controller and plant matrices. In these special cases, based on the LMI formulation, the minimization of the performance level can be carried out as a simple convex optimization problem that converges to a global minimum. Section III-A proposes a LMI to ascertain the performance of a given antiwindup compensator applied to a given system. In Section III-C, it is shown that, once the necessary and sufficient conditions have been verified, it is possible to construct the desired antiwindup compensator by solving another LMI which efficiently provides a state-space representation of the dynamics of such an antiwindup compensator. In Section IV, the proposed antiwindup construction method is applied to a simulation example taken from the literature and to an experimental system. The remaining Section $\mathrm{V}$ provides the necessary tools for the proof of the main contribution of this paper through the statement and proof of interesting intermediate results.

\section{PRoblem Definition}

\section{A. Unconstrained Closed-Loop System}

Consider a linear plant given by

$$
\mathcal{P}\left\{\begin{array}{l}
\dot{x}_{p}=A_{p} x_{p}+B_{p, u} u+B_{p, w} w \\
y=C_{p, y} x_{p}+D_{p, y u} u+D_{p, y w} w \\
z=C_{p, z} x_{p}+D_{p, z u} u+D_{p, z w} w
\end{array}\right.
$$

where $x_{p} \in \mathbb{R}^{n_{p}}$ is the plant state, $u \in \mathbb{R}^{n_{u}}$ is the control input, $w \in \mathbb{R}^{n_{w}}$ is the exogenous input (possibly containing disturbance, reference and measurement noise), $y \in \mathbb{R}^{n_{y}}$ is the plant output available for measurement, $z \in \mathbb{R}^{n_{z}}$ is the performance output (possibly corresponding to a weighted tracking error) and $A_{p}, B_{p, u}, B_{p, w}, C_{p, y}, D_{p, y u}, D_{p, y w}, C_{p, z}, D_{p, z u}$, and $D_{p, z w}$ are matrices of suitable dimensions. The plant with $u \equiv 0$ will be referred to as the open-loop plant.

Assume also that, an unconstrained controller has been designed

$$
\mathcal{C}\left\{\begin{array}{l}
\dot{x}_{c}=A_{c} x_{c}+B_{c, y} y+B_{c, w} w+v_{1} \\
y_{c}=C_{c} x_{c}+D_{c, y} y+D_{c, w} w+v_{2}
\end{array}\right.
$$

(where $x_{c} \in \mathbb{R}^{n_{c}}$ is the controller state, $y_{c} \in \mathbb{R}^{n_{u}}$ is the controller output, $v_{1}$ and $v_{2}$ are additional inputs that will be used for antiwindup augmentation and $A_{c}, B_{c, y}, B_{c, w}, C_{c}, D_{c, y}$, and $D_{c, w}$ are matrices of suitable dimensions) in such a way that its interconnection to the linear plant through the equations

$$
u=y_{c} \quad v_{1}=0 \quad v_{2}=0
$$

is well-posed and guarantees internal stability of the arising closed-loop system. The interconnection of (1) and (2) via (3) corresponds to the block diagram in Fig. 2 which we will refer to as the unconstrained closed-loop system. By selecting the state $x_{\ell}:=\left[\begin{array}{ll}x_{p}^{T} & x_{c}^{T}\end{array}\right]^{T} \in \mathbb{R}^{n_{\mathrm{CL}} \times n_{\mathrm{CL}}}$, where $n_{\mathrm{CL}}:=n_{p}+n_{c}$, and focusing on the effect of the exogenous input $w$ on the performance output $z$, we can write the dynamics of the unconstrained closed-loop system as a single linear system with state-space representation

$$
\begin{aligned}
\dot{x}_{\ell} & =A_{\mathrm{CL}} x_{\ell}+B_{\mathrm{CL}, w} w \\
z & =C_{\mathrm{CL}, z} x_{\ell}+D_{\mathrm{CL}, z w} w
\end{aligned}
$$

where $A_{\mathrm{CL}}, B_{\mathrm{CL}, w}, C_{\mathrm{CL}, z}$, and $D_{\mathrm{CL}, z w}$ are uniquely determined by the matrices in (1) and (2).

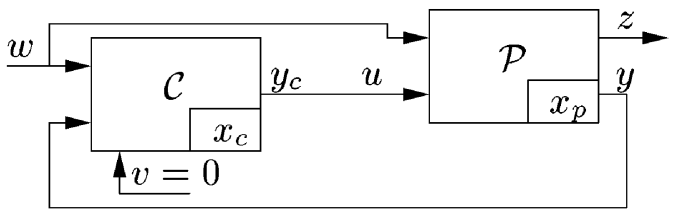

Fig. 2. Unconstrained closed-loop system.

\section{B. Input Saturation and Antiwindup Augmentation}

Instead of considering a particular plant input nonlinearity, we consider a class of input nonlinearities defined in Definition 2 (which requires the immediately following definition) in order to state necessary and sufficient conditions for stability and performance.

Definition 1: Given any symmetric positive-definite matrix $V_{s} \in \mathbb{R}^{n_{u} \times n_{u}}$ and two matrices $W_{1}, W_{2} \in \mathbb{R}^{n_{u} \times r}$, define the $V_{s}$-product of $W_{1}$ and $W_{2}$ as

$$
\left\langle W_{1}, W_{2}\right\rangle_{V_{s}}:=W_{1}^{T} V_{s} W_{2} \text {. }
$$

A function $f: \mathbb{R}^{n_{u}} \rightarrow \mathbb{R}^{n_{u}}$ is said to belong to the sector $[0, I]_{V_{s}}$ if $\langle f(w), w-f(w)\rangle_{V_{s}} \geq 0$ for all $w \in \mathbb{R}^{n_{u}}$. A function $f: \mathbb{R}^{n_{u}} \rightarrow \mathbb{R}^{n_{u}}$ is said to belong to the incremental sector $[0, I]_{V_{s}}$ if $\langle J f(y), I-J f(y)\rangle_{V_{s}} \geq 0$ for almost all $y \in \mathbb{R}^{n_{u}}$, where $J f(y)$ denotes the Jacobian of $f$ evaluated at $y$.

Definition 2: A function $\phi: \mathbb{R}^{n_{u}} \rightarrow \mathbb{R}^{n_{u}}$ is said to belong to $\Phi_{V_{s}}$ if the function $\phi(\cdot)$ is locally Lipschitz, belongs to the incremental sector $[0, I]_{V_{s}}$ and $\phi(0)=0$.

Remark 1: If $\phi(\cdot)$ belongs to $\Phi_{V_{s}}$ then $\phi(\cdot)$ belongs to the sector $[0, I]_{V_{s}}$. Also, when $V_{s}=I$, the $V_{s}$-product $\left\langle W_{1}, W_{2}\right\rangle_{V_{s}}$ coincides with the standard product $W_{1}^{T} W_{2}$. Furthermore, the sector $[0, I]_{I}$ property coincides with the sector $[0, I]$ property defined in [14, p. 403].

Suppose the control input of the plant is subject to a nonlinearity, namely

$$
u=\phi\left(y_{c}\right)
$$

where $\phi(\cdot)$ belongs to $\Phi_{V_{s}}$.

Remark 2: The $\phi(\cdot)$ in (5) could be a decentralized saturation function, namely

$$
\operatorname{sat}\left(y_{c}\right):=\left[\begin{array}{llll}
\operatorname{sat}_{1}\left(y_{c 1}\right) & \operatorname{sat}_{2}\left(y_{c 2}\right) & \cdots & \operatorname{sat}_{n_{u}}\left(y_{c n_{u}}\right)
\end{array}\right]^{T}
$$

where $^{1}$

$$
\operatorname{sat}_{i}\left(y_{c i}\right):=\frac{y_{c i}}{\max \left\{1, \frac{\left|y_{c i}\right|}{M_{i}}\right\}}
$$

$M_{i} \in \mathbb{R}, M_{i}>0$ for $i=1, \ldots, n_{u}$. Such decentralized saturation functions belong to $\Phi_{V_{s}}$ if $V_{s}$ is a diagonal positive-definite matrix.

Given an integer $n_{\text {aw }} \geq 0$, we address the problem of designing an order $n_{\text {aw }}$ linear antiwindup compensator

$$
\mathcal{A W}\left\{\begin{array}{l}
\dot{x}_{\mathrm{aw}}=\Lambda_{1} x_{\mathrm{aw}}+\Lambda_{2}\left(y_{c}-u\right) \\
v=\left[\begin{array}{l}
v_{1} \\
v_{2}
\end{array}\right]=\Lambda_{3} x_{\mathrm{aw}}+\Lambda_{4}\left(y_{c}-u\right)
\end{array}\right.
$$

${ }^{1}$ For the purpose of this paper, decentralized saturation can denote the larger set of decentralized functions where $\operatorname{sat}_{i}(\cdot)$ is locally Lipschitz, $\operatorname{sat}_{i}(0)=0$ and $(d /(d s)) \operatorname{sat}_{i}(s) \in[0,1]$ almost everywhere for $i=1, \ldots, n_{u}$. 


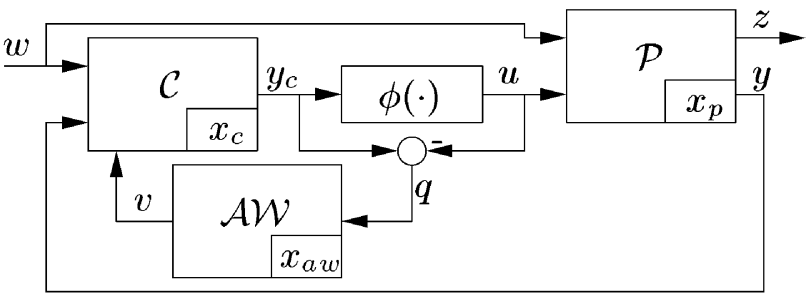

Fig. 3. Antiwindup closed-loop system.

(where $x_{\text {aw }} \in \mathbb{R}^{n_{\text {aw }}}$ is the antiwindup state, $v \in \mathbb{R}^{n_{v}}$ (with $\left.n_{v}:=n_{c}+n_{u}\right)$ is the antiwindup output, and the matrices $\Lambda_{1}, \Lambda_{2}, \Lambda_{3}$, and $\Lambda_{4}$ are of suitable dimensions) that guarantees a desirable $\mathcal{L}_{2}$ relationship between the exogenous input $w$ and the performance output $z$ for all $\phi(\cdot)$ that belong to $\Phi_{V_{s}}$. The interconnection (1), (2), (5), (6) will henceforth be called the antiwindup closed-loop system and is shown in Fig. 3.

\section{Lyapunov Characterization of Stability and Performance}

A desirable stability and performance property for the antiwindup closed-loop system will be presented in terms of Lyapunov analysis tools.

Definition 3: Given the linear plant $\mathcal{P}$ in (1) and the unconstrained controller $\mathcal{C}$ in (2), a linear antiwindup compensator (6) of order $n_{\text {aw }}$ guarantees well-posedness and quadratic performance of level $\gamma$ if the augmented antiwindup closed-loop system (1), (2), (5), (6) is such that, for all $\phi(\cdot)$ that belong to $\Phi_{V_{s}}$

1) the interconnection (1), (2), (5), (6) is well-posed;

2) there exists a scalar $\epsilon>0$ and a quadratic Lyapunov function $V(x)=x^{T} P x$ (with $x:=\left[\begin{array}{lll}x_{p}^{T} & x_{c}^{T} & x_{\mathrm{aw}}^{T}\end{array}\right]^{T}$ and $P=P^{T}>0$ ) such that its time derivative $\dot{V}$ along the dynamics of (1), (2), (5), (6) satisfies

$$
\dot{V}<-\epsilon x^{T} x-\frac{1}{\gamma} z^{T} z+\gamma w^{T} w \quad \forall(x, w) \neq 0 .
$$

Remark 3: Definition 3 entails (sufficient) conditions for internal stability of the antiwindup closed-loop system and for finite $\mathcal{L}_{2}$ gain $\gamma$ from $w$ to $z$ for all $\phi(\cdot)$ that belong to $\Phi_{V_{s}}$. Indeed, since the interconnection (5) is well-posed [as guaranteed by item 1)], item 2) guarantees

i) quadratic stability, derived by rewriting (7) with $w=0$, which implies

$$
\dot{V} \leq-\epsilon|x|^{2}
$$

ii) $\mathcal{L}_{2}$ gain from $w$ to $z$ smaller than $\gamma$. Indeed, inequality (7) can be integrated on both sides from 0 to $t$ (assuming zero initial conditions) to obtain

$0 \leq V(t)+\epsilon \int_{0}^{t}|x|^{2} d t^{\prime} \leq \frac{-1}{\gamma} \int_{0}^{t}|z|^{2} d t^{\prime}+\gamma \int_{0}^{t}|w|^{2} d t^{\prime}$

which implies the finite $\mathcal{L}_{2}$ gain $\gamma$ from $w$ to $z$ :

$$
\|z\|_{2} \leq \gamma\|w\|_{2}
$$

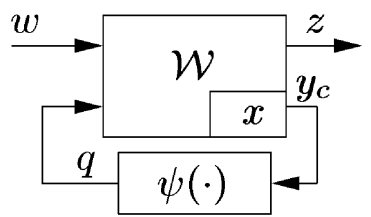

Fig. 4. Compact antiwindup closed-loop system.

\section{LMI-BASED ANTIWINDUP ANALYSIS AND SYNTHESIS}

The main contribution of this paper is presented in three parts. In Section III-A, we will provide tools for performance analysis when the antiwindup augmentation (6) is preassigned. In Section III-B, we provide nonlinear matrix conditions whose feasibility is necessary and sufficient to guarantee the existence of an antiwindup compensator that guarantees stability and performance in the sense of Definition 3. For special cases, these nonlinear matrix conditions are transformed into a set of LMIs. Finally, in Section III-C, we will give a procedure to construct antiwindup compensators that induce the performance levels guaranteed by suitable solutions to the matrix conditions in Section III-B.

\section{A. LMI-Based Antiwindup Performance Analysis}

Assume that the plant $\mathcal{P}$ in (1), the controller $\mathcal{C}$ in (2) and the linear antiwindup compensator $\mathcal{A W}$ in (6) are given. Then, for analysis purposes, the level of performance can be determined by solving an LMI eigenvalue problem ${ }^{2}$.

To formulate suitably the corresponding LMIs, we need to introduce additional notation which corresponds to representing the antiwindup closed-loop system in a compact way, as in Fig. 4. In particular define $\psi(\cdot): \mathbb{R}^{n_{u}} \rightarrow \mathbb{R}^{n_{u}}$ with output $q \in \mathbb{R}^{n_{u}}$ as

$$
q=\psi\left(y_{c}\right):=y_{c}-\phi\left(y_{c}\right) .
$$

Next, define the overall state variable $x \in \mathbb{R}^{n}$, where $n:=$ $n_{p}+n_{c}+n_{\text {aw }}$, as

$$
x:=\left[\begin{array}{lll}
x_{p}^{T} & x_{c}^{T} & x_{\mathrm{aw}}^{T}
\end{array}\right]^{T}
$$

which allows the linear dynamics of the plant, controller and antiwindup compensator to be combined and written as

$$
\mathcal{W}\left\{\begin{array}{l}
\dot{x}=A x+B_{q} q+B_{w} w \\
y_{c}=C_{y} x+D_{y q} q+D_{y w} w \\
z=C_{z} x+D_{z q} q+D_{z w} w
\end{array}\right.
$$

where the matrices $A, B_{q}, B_{w}, C_{y}, D_{y q}, D_{y w}, C_{z}, D_{z q}$, and $D_{z w}$ are of appropriate dimensions and are uniquely determined by the matrices in (1), (2), and (6).

After a suitable change of coordinates the interconnection between (8) and (9), named the compact antiwindup closed-loop system and shown in Fig. 4, corresponds to the antiwindup closed-loop system (1), (2), (5), (6).

Theorem 1: Given the antiwindup closed-loop system (8), (9) and a scalar $\tilde{\gamma}$, the antiwindup closed-loop system is wellposed and guarantees quadratic performance of level $\tilde{\gamma}$ if and

${ }^{2}$ The LMI eigenvalue problem (see, e.g., [4, p. 10]) is to minimize a linear function subject to an LMI constraint (or to determine that the constraint is unfeasible). 
only if there exists a solution $(Q, \delta, \gamma)$ to the following LMI problem:

$$
\left[\begin{array}{cccc}
Q A^{T}+A Q & B_{q} U+Q C_{y}^{T} & B_{w} & Q C_{z}^{T} \\
U B_{q}^{T}+C_{y} Q & D_{y q} U+U D_{y q}^{T}-2 U & D_{y w} & U D_{z q}^{T} \\
B_{w}^{T} & D_{y w}^{T} & -\gamma I & D_{z w}^{T} \\
C_{z} Q & D_{z q} U & D_{z w} & -\gamma I
\end{array}\right]<0
$$

$Q=Q^{T}>0$

$U=\delta V_{s}^{-1}>0$

$\gamma \leq \tilde{\gamma}$

Proof: See Section V-A.

Remark 4: Convex Performance Analysis: Given a plant, controller and antiwindup compensator that make up an antiwindup closed-loop system, the greatest lower bound on performance $\tilde{\gamma}^{*}$ can be obtained by solving in the unknowns $(Q, \delta, \gamma)$ the convex LMI eigenvalue problem $\tilde{\gamma}^{*}:=\inf (\gamma)$ subject to (10a)-(10c).

Remark 5: If $\phi(\cdot)$ belongs to $\Phi_{V_{s}}$ and $V_{s}^{-1}$ is linearly parameterized, then extra degrees of freedom can be exploited when solving the LMIs (10). This is the case for decentralized saturation functions introduced in Remark 2. Observe that $\delta V_{s}^{-1}$ is linearly parameterized over the family of diagonal positive definite matrices. Hence, in the decentralized case, (10c) can be replaced by $U=\operatorname{diag}\left(u_{1}, \ldots u_{n_{u}}\right)>0$ where $u_{i}$ are unknown, thus allowing extra degrees of freedom in the minimization of $\gamma$.

Although Theorem 1 provides a useful tool for analysis purposes, it can not easily be used for antiwindup synthesis because the unknown antiwindup compensator matrices multiply the unknown $Q$, thus making the matrix inequality (10a) nonlinear. In the sequel, suitable procedures are given to construct antiwindup compensators that guarantee well-posedness and quadratic performance.

\section{B. Feasibility of the Antiwindup Synthesis Problem}

To assist in the system theoretic interpretation of the matrix inequalities that will follow, recall the well-known LMI formulation of the bounded real lemma for continuous time systems (for a complete proof see, e.g., [25, p. 82]).

Lemma 1 (Bounded Real Lemma): The following statements are equivalent.

1) $\left\|D+C(s I-A)^{-1} B\right\|_{\infty}<\gamma$ and $A$ is Hurwitz.

2) There exists a symmetric positive-definite solution $X$ to the LMI

$$
\left[\begin{array}{ccc}
X A^{T}+A X & B & X C^{T} \\
B^{T} & -\gamma I & D^{T} \\
C X & D & -\gamma I
\end{array}\right]<0
$$

The following definition will be useful to simplify the notation throughout this paper.

Definition 4: Given the plant $\mathcal{P}$ in (1), the controller $\mathcal{C}$ in (2), an integer $n_{\text {aw }} \geq 0$ and a scalar $\tilde{\gamma}$, define the matrix conditions
$\operatorname{MC}\left(\mathcal{P}, \mathcal{C}, n_{\text {aw }}, \tilde{\gamma}\right)$ as the following set of matrix conditions in the unknowns $(R, S, \gamma)$ :

$$
\begin{aligned}
& {\left[\begin{array}{ccc}
R_{11} A_{p}^{T}+A_{p} R_{11} & B_{p, w} & R_{11} C_{p, z}^{T} \\
B_{p, w}^{T} & -\gamma I & D_{p, z w}^{T} \\
C_{p, z} R_{11} & D_{p, z w} & -\gamma I
\end{array}\right]<0} \\
& {\left[\begin{array}{ccc}
S A_{\mathrm{CL}}^{T}+A_{\mathrm{CL}} S & B_{\mathrm{CL}, w} & S C_{\mathrm{CL}, z}^{T} \\
B_{\mathrm{CL}, w}^{T} & -\gamma I & D_{\mathrm{CL}, z w}^{T} \\
C_{\mathrm{CL}, z} S & D_{\mathrm{CL}, z w} & -\gamma I
\end{array}\right]<0} \\
& R=R^{T}=\left[\begin{array}{ll}
R_{11} & R_{12} \\
R_{12}^{T} & R_{22}
\end{array}\right]>0 \\
& S=S^{T}>0 \\
& R-S \geq 0 \\
& \operatorname{rank}(R-S) \leq n_{\mathrm{aw}} \\
& \gamma \leq \tilde{\gamma} .
\end{aligned}
$$

Moreover, $\operatorname{MC}\left(\mathcal{P}, \mathcal{C}, n_{\text {aw }}, \tilde{\gamma}\right)$ is said to be feasible if there exists a solution $(R, S, \gamma)$ that satisfies (11).

The following theorem, representing our main result, provides necessary and sufficient conditions for the existence of an antiwindup compensator that guarantees well-posedness and quadratic performance of level $\tilde{\gamma}$ in terms of the matrix conditions $\mathrm{MC}\left(\mathcal{P}, \mathcal{C}, n_{\text {aw }}, \tilde{\gamma}\right)$.

Theorem 2: Given the plant $\mathcal{P}$ in (1), the unconstrained controller $\mathcal{C}$ in (2), an integer $n_{\text {aw }} \geq 0$ and scalar $\tilde{\gamma}$, there exists a linear antiwindup compensator of order $n_{\text {aw }}$ that guarantees well-posedness and quadratic performance of level $\tilde{\gamma}$ if and only if $\operatorname{MC}\left(\mathcal{P}, \mathcal{C}, n_{\text {aw }}, \tilde{\gamma}\right)$ is feasible.

Proof: See Section V.

Remark 6: The Greatest Lower Bound on Achievable Performance: The goal of optimal antiwindup design is to construct an antiwindup compensator that guarantees a performance level as small as possible. Based on Theorem 2, the greatest lower bound on achievable performance $\tilde{\gamma}^{*}$ such that $\operatorname{MC}\left(\mathcal{P}, \mathcal{C}, n_{\text {aw }}, \tilde{\gamma}^{*}\right)$ is feasible can, in principle, be determined by solving in the unknowns $(R, S, \gamma)$ the nonconvex optimization problem $\tilde{\gamma}^{*}:=\inf (\gamma)$ subject to (11a)-(11f).

Remark 7 Lower Bounds on Performance Level: Using Lemma 1, (11a) and (11b) have a system theoretic interpretation. In particular, observe that (11a) constrains $\gamma$ to be no less than the $\mathcal{H}_{\infty}$ norm of the plant $\mathcal{P}$ with $u \equiv 0$, input $w$ and output $z$ or equivalently, no less than the $\mathcal{L}_{2}$ gain from $w$ to $z$ associated with the open-loop plant. Similarly, (11b) constrains $\gamma$ to be no less than the $\mathcal{L}_{2}$ gain of the unconstrained closed-loop system (4). While these two LMIs provide lower bounds for the $\mathcal{L}_{2}$ gain achievable by the antiwindup closed-loop system, (11e) and (11f) establish a nonlinear coupling between the two conditions.

Based on the previous remark, it is evident that for condition (11a) to be feasible the plant (1) needs to be asymptotically stable. Since Theorem 2 also establishes the necessity of (11) for antiwindup feasibility, asymptotic stability of the plant is shown there to be necessary if one wants to guarantee the global properties of Definition 3. One of the reasons that it is necessary for $A_{p}$ to be Hurwitz is that we are asking for global quadratic stability in the absence of inputs. Even if we didn't insist on quadratic 
stability, with appropriate detectability and stabilizability conditions from $w$ to $z$, it is a straightforward consequence of the classical small gain theorem that finite gain $\mathcal{L}_{2}$ stabilizability by bounded controls implies that $A_{p}$ is Hurwitz. In the more general case of non asymptotically stable linear plants (which is not addressed in this paper), the global properties of Definition 3 should be relaxed to be able to guarantee useful results.

In the next section, we will show that the nonlinear condition (11f) can be transformed into a linear one, in some special cases.

1) LMI Formulations of the Feasibility Condition: An appealing property of Theorem 2 is that all but one of the conditions in $\operatorname{MC}\left(\mathcal{P}, \mathcal{C}, n_{\text {aw }}, \tilde{\gamma}\right)$ are linear with respect to the unknowns $(R, S, \gamma)$, the exception being (11f) - the rank condition. Paralleling the necessary and sufficient conditions for reduced order $\mathcal{H}_{\infty}$ control synthesis (see, e.g., [8, eq. (26)], when considering the full order case $n_{\text {aw }}=n_{p}+n_{c}$, the rank condition is trivially satisfied and the optimization of the performance level and the determination of the corresponding solution $(R, S, \gamma)$ reduces to a convex LMI eigenvalue problem, for which numeric algorithms are readily available (see, e.g., [9]).

For the full-order case, the rank condition is guaranteed satisfied and the optimal performance level $\tilde{\gamma}^{*}$ such that $\operatorname{MC}\left(\mathcal{P}, \mathcal{C}, n_{p}+n_{c}, \tilde{\gamma}^{*}\right)$ is feasible can be determined by solving in the unknowns $(R, S, \gamma)$ the LMI eigenvalue problem $\tilde{\gamma}^{*}:=\inf (\gamma)$ subject to (11a)-(11e). However, when considering antiwindup compensation of reduced-order $\left(n_{\text {aw }}<n_{p}+n_{c}\right)$, the rank condition needs to be satisfied and the conditions $\operatorname{MC}\left(\mathcal{P}, \mathcal{C}, n_{\mathrm{aw}}, \tilde{\gamma}\right)$ become nonlinear. By exploiting the special structure of the antiwindup design problem, in the following Propositions 1 and 2 we will show how to replace the nonlinear rank condition with equivalent linear conditions, for the special reduced order cases $n_{\mathrm{aw}}=0$ and $n_{\text {aw }} \geq n_{p}$, respectively. In these two special cases, all the matrix inequalities are linear in the unknowns, and the minimization problem for $\tilde{\gamma}$ becomes a convex LMI eigenvalue problem.

Proposition $1\left(n_{\text {aw }}=0\right)$ : Given the plant $\mathcal{P}$ in (1), the controller $\mathcal{C}$ in (2) and a scalar $\tilde{\gamma}, \operatorname{MC}(\mathcal{P}, \mathcal{C}, 0, \tilde{\gamma})$ is feasible if and only if there exists a solution $(R, \gamma)$ to the following LMI conditions:

$$
\begin{aligned}
& {\left[\begin{array}{ccc}
R_{11} A_{p}^{T}+A_{p} R_{11} & B_{p, w} & R_{11} C_{p, z}^{T} \\
B_{p, w}^{T} & -\gamma I & D_{p, z w}^{T} \\
C_{p, z} R_{11} & D_{p, z w} & -\gamma I
\end{array}\right]<0} \\
& {\left[\begin{array}{ccc}
R A_{\mathrm{CL}}^{T}+A_{\mathrm{CL}} R & B_{\mathrm{CL}, w} & R C_{\mathrm{CL}, z}^{T} \\
B_{\mathrm{CL}, w}^{T} & -\gamma I & D_{\mathrm{CL}, z w}^{T} \\
C_{\mathrm{CL}, z} R & D_{\mathrm{CL}, z w} & -\gamma I
\end{array}\right]<0} \\
& R=R^{T}=\left[\begin{array}{ll}
R_{11} & R_{12} \\
R_{12}^{T} & R_{22}
\end{array}\right]>0 \\
& \gamma \leq \tilde{\gamma} .
\end{aligned}
$$

Proof: If $n_{\text {aw }}=0$, (11f) is satisfied if and only if $R=S$; thus (11e) is satisfied and (11d) is redundant. Hence, the proof follows by rewriting the remaining inequalities in (11) with $R=$ $S$.

Proposition $2\left(n_{\mathrm{aw}} \geq n_{p}\right)$ : Given the plant $\mathcal{P}$ in (1), the controller $\mathcal{C}$ in (2), an integer $n_{\text {aw }} \geq n_{p}$ and a scalar $\tilde{\gamma}, \operatorname{MC}\left(\mathcal{P}, \mathcal{C}, n_{\text {aw }}, \tilde{\gamma}\right)$ is feasible if and only if there exists a solution $\left(R_{11}, S, \gamma\right)$ to the following LMI problem:

$$
\begin{aligned}
& {\left[\begin{array}{ccc}
R_{11} A_{p}^{T}+A_{p} R_{11} & B_{p, w} & R_{11} C_{p, z}^{T} \\
B_{p, w}^{T} & -\gamma I & D_{p, z w}^{T} \\
C_{p, z} R_{11} & D_{p, z w} & -\gamma I
\end{array}\right]<0} \\
& {\left[\begin{array}{ccc}
S A_{\mathrm{CL}}^{T}+A_{\mathrm{CL}} S & B_{\mathrm{CL}, w} & S C_{\mathrm{CL}, z}^{T} \\
B_{\mathrm{CL}, w}^{T} & -\gamma I & D_{\mathrm{CL}, z w}^{T} \\
C_{\mathrm{CL}, z} S & D_{\mathrm{CL}, z w} & -\gamma I
\end{array}\right]<0} \\
& R_{11}=R_{11}^{T}>0 \\
& S=S^{T}=\left[\begin{array}{ll}
S_{11} & S_{12} \\
S_{12}^{T} & S_{22}
\end{array}\right]>0 \\
& R_{11}-S_{11}>0 \\
& \gamma \leq \tilde{\gamma} .
\end{aligned}
$$

Proof [Feasibility of (13) $\Rightarrow$ Feasibility of (11)]: Given a solution $\left(R_{11}, S, \gamma\right)$ to (13), take $R_{12}=S_{12}$ and $R_{22}=S_{22}$. Then $R$ and $S$ trivially satisfy the rank constraint (11f) since, by (13e), $R_{11}>S_{11}$, then $R \geq S>0$. Hence, $R$ is positive definite and $(R, S, \gamma)$ satisfies Conditions (11) with $n_{\text {aw }} \geq n_{p}$.

[Feasibility of (11) $\Rightarrow$ Feasibility of (13)]. Suppose (11) is satisfied by a solution $(R, S, \gamma)$. Then (11e) guarantees $R_{11}-$ $S_{11} \geq 0$. Then there exists a symmetric positive-definite matrix $\bar{R}_{11}$, such that with $\bar{R}_{11}-S_{11}>0$, (13a) is satisfied. ([To show this, take $\epsilon>0$ such that $\bar{R}_{11}=R_{11}+\epsilon I_{n_{p}}$ satisfies (13a). Moreover, $R_{11}-S_{11} \geq 0 \Rightarrow \bar{R}_{11}-\epsilon I_{n_{p}}-S_{11} \geq$ $0 \Rightarrow \bar{R}_{11}-S_{11}>0$, as desired]. Finally, (13) is satisfied by $\left(\bar{R}_{11}, S, \gamma\right)$.

Based on Theorem 2 and Propositions 1 and 2, the following theorem gives suitable conditions for the feasibility of the conditions $\operatorname{MC}\left(\mathcal{P}, \mathcal{C}, n_{\text {aw }}, \tilde{\gamma}\right)$ in Definition 4.

Theorem 3: The following properties hold.

1) There exists a scalar $\tilde{\gamma}$ such that $\operatorname{MC}(\mathcal{P}, \mathcal{C}, 0, \tilde{\gamma})$ is feasible if and only if there exists a matrix $R$ that is a solution to the LMI problem

$$
\begin{array}{r}
R_{11} A_{p}^{T}+A_{p} R_{11}<0 \\
R A_{\mathrm{CL}}^{T}+A_{\mathrm{CL}} R<0 \\
R=R^{T}=\left[\begin{array}{ll}
R_{11} & R_{12} \\
R_{12}^{T} & R_{22}
\end{array}\right]>0 .
\end{array}
$$

2) There exists a scalar $\tilde{\gamma}$ such that $\operatorname{MC}\left(\mathcal{P}, \mathcal{C}, n_{p}, \tilde{\gamma}\right)$ is feasible if and only if $A_{p}$ is Hurwitz.

3) If $\operatorname{MC}\left(\mathcal{P}, \mathcal{C}, n_{1}, \tilde{\gamma}\right)$ is feasible and $n_{1} \leq n_{2}$, then $\operatorname{MC}\left(\mathcal{P}, \mathcal{C}, n_{2}, \tilde{\gamma}\right)$ is feasible.

4) If $\operatorname{MC}\left(\mathcal{P}, \mathcal{C}, n_{1}, \tilde{\gamma}\right)$ is feasible and $n_{1} \geq n_{p}$, then $\operatorname{MC}\left(\mathcal{P}, \mathcal{C}, n_{p}, \tilde{\gamma}\right)$ is feasible.

Proof:

Item 1) If $\operatorname{MC}(\mathcal{P}, \mathcal{C}, 0, \tilde{\gamma})$ is feasible then by Proposition 1 there exists a matrix $R$ that satisfies (12a) and (12b) with $\gamma=\tilde{\gamma}$. Since each block on the main diagonal of both (12a) and (12b) is negative definite, then the top left block diagonal entries which correspond to the inequalities (14), are negative definite as well.

Assume there exists a symmetric positive definite matrix $R$ that satisfies (14). Since (14) corresponds to the top left block diagonal entries of Conditions (12a) and (12b), then there exists a large enough $\gamma=\bar{\gamma}$ such 
that (12a) and (12b) are satisfied. The result follows from Proposition 1 picking $\tilde{\gamma}=\bar{\gamma}$.

Item 2) First, note that there exists a matrix $R_{11}=R_{11}^{T}>0$ such that $R_{11} A_{p}^{T}+A_{p} R_{11}<0$ if and only if $A_{p}$ is Hurwitz. Moreover, since the unconstrained closed-loop system is exponentially stable, $A_{\mathrm{CL}}$ is Hurwitz and there exists a matrix $\bar{S}=\bar{S}^{T}>0$ such that $\bar{S} A_{\mathrm{CL}}^{T}+A_{\mathrm{CL}} \bar{S}<0$. Since $R_{11}>0$, there exists a sufficiently small $\epsilon>0$ such that $R_{11}-\epsilon \bar{S}_{11}>0$. Take $S=\epsilon \bar{S}$. Then there exists a large enough $\gamma=\bar{\gamma}$ such that $\left(R_{11}, S, \gamma\right)$ satisfies (13). The proof is completed by applying Proposition 2 picking $\tilde{\gamma}=\bar{\gamma}$.

Item 3) The result is a direct consequence of Definition 4 since if the rank condition (11f) holds for $n_{\text {aw }}=$ $n_{1}$ then it also holds for $n_{\text {aw }}=n_{2} \geq n_{1}$.

Item 4) The result is a direct consequence of Proposition 2 since Conditions (13) are independent of $n_{\mathrm{aw}}$.

An important implication of Theorem 3 is that not only does the antiwindup construction always admit a solution choosing $n_{\text {aw }}=n_{p}$, but also given the optimal performance $\gamma^{*}$ achievable by a solution of any order $n_{\text {aw }} \geq n_{p}$, then by item 4 of the theorem, this same performance is achievable by an antiwindup compensator of order $n_{p}$. Hence, the restriction that the antiwindup compensator order is $n_{p}$ does not restrict the minimum achievable performance level.

Moreover, item 1) of Theorem 3 implies that, in many situations, static antiwindup compensation does not provide a feasible solution to this antiwindup problem, regardless of the performance level $\tilde{\gamma}$. Indeed, condition (14) corresponds to requiring the existence of a quasi-common quadratic Lyapunov function between the open-loop plant and the unconstrained closed-loop system. In particular, if the unconstrained controller is static $\left(R_{11}=R\right)$, it exactly requires a common quadratic Lyapunov function. In the general case of a dynamic unconstrained controller, it is a generalization of this requirement based on the fact that the size of the unconstrained closed-loop system is larger than the size of the open-loop plant.

Remark 8: Greatest Lower Bound on Achievable Performance via Convex Optimization: Remark 6 provides a method to determine the greatest lower bound on performance by solving a nonconvex optimization problem. In the light of Propositions 1 and 2, the greatest lower bound on performance can be determined by solving a convex optimization problem when considering static or at least plant-order antiwindup compensation. In particular, the greatest lower bound on achievable performance, $\tilde{\gamma}_{s}^{*}$, using a static antiwindup compensator can be determined by solving, in the unknowns $(R, \gamma)$, the convex LMI eigenvalue problem: $\tilde{\gamma}_{s}^{*}:=\inf (\gamma)$ subject to $(12 \mathrm{a})-(12 \mathrm{c})$. Similarly, the greatest lower bound on achievable performance, $\tilde{\gamma}_{n_{p}}^{*}$, using an antiwindup compensator of order greater than or equal to the order of the plant can be determined by solving, in the unknowns $\left(R_{11}, S, \gamma\right)$, the convex LMI eigenvalue problem: $\tilde{\gamma}_{n_{p}}^{*}:=\inf (\gamma)$ subject to $(13 \mathrm{a})-(13 \mathrm{e})$.

\section{LMI-Based Antiwindup Synthesis}

Although the results in Section III-B provide natural conditions for the existence of an antiwindup compensator achieving a certain performance level for the closed-loop system in Fig. 3, they do not provide tools for the construction of such a compensator. In this section, based on a solution $(R, S, \gamma)$ to $\operatorname{MC}\left(\mathcal{P}, \mathcal{C}, n_{\text {aw }}, \tilde{\gamma}\right)$ arising from Theorem 2 or Proposition 1 or 2 , we give a procedure to construct a state-space representation of an antiwindup compensator that guarantees well-posedness and quadratic performance of level $\tilde{\gamma}$. The effectiveness of the procedure is then formally stated in Theorem 4.

To suitably describe the procedure for the construction of the antiwindup compensator, we will first introduce an equivalent representation for the antiwindup closed-loop system (1), (2), (5), (6) represented in Fig. 3. By stacking the plant and the controller states into a single state vector $x_{\mathrm{CL}}:=\left[\begin{array}{ll}x_{p}^{T} & x_{c}^{T}\end{array}\right]^{T} \in$ $\mathbb{R}^{n_{\mathrm{CL}}}$, with $n_{\mathrm{CL}}:=n_{p}+n_{c}$, the antiwindup closed-loop system can be written as shown in Fig. 5. The dynamics of the subsystem $\mathcal{H}$ in Fig. 5 is given by

$$
\mathcal{H}\left\{\begin{array}{l}
\dot{x}_{\mathrm{CL}}=A_{\mathrm{CL}} x_{\mathrm{CL}}+B_{\mathrm{CL}, w} w+B_{\mathrm{CL}, q} q+B_{\mathrm{CL}, v} v \\
z=C_{\mathrm{CL}, z} x_{\mathrm{CL}}+D_{\mathrm{CL}, z w} w+D_{\mathrm{CL}, z q} q+D_{\mathrm{CL}, z v} v \\
y_{c}=C_{\mathrm{CL}, y} x_{\mathrm{CL}}+D_{\mathrm{CL}, y w} w+D_{\mathrm{CL}, y q} q+D_{\mathrm{CL}, y v} v
\end{array}\right.
$$

where the matrices $A_{\mathrm{CL}}, B_{\mathrm{CL}, w}, B_{\mathrm{CL}, q}, B_{\mathrm{CL}, v}$, $C_{\mathrm{CL}, z}, D_{\mathrm{CL}, z w}, D_{\mathrm{CL}, z q}, D_{\mathrm{CL}, z v}, C_{\mathrm{CL}, y}, \quad D_{\mathrm{CL}, y w}, D_{\mathrm{CL}, y q}$, and $D_{\mathrm{CL}, y v}$ are of appropriate dimensions and only depend on the matrices of the plant (1) and of the controller (2).

Based on the linear system (15), we can formalize a procedure for the construction of the antiwindup compensator.

1) Procedure 1 (Construction of the Antiwindup Compensator):

Step 1) Solve the feasibility conditions.

Given the plant $\mathcal{P}$, the controller $\mathcal{C}$, an integer $n_{\text {aw }} \geq 0$ and a scalar $\tilde{\gamma}$, determine a solution $(R, S, \gamma)$ that satisfies the conditions $\operatorname{MC}\left(\mathcal{P}, \mathcal{C}, n_{\text {aw }}, \tilde{\gamma}\right)$

Step 2) Construct the matrix $Q$.

Using the solution $(R, S, \gamma)$ from Step 1, define the matrix $N \in \mathbb{R}^{n_{\mathrm{CL}} \times n_{\text {aw }}}$ as a solution of the following equation:

$$
R S^{-1} R-R=N N^{T} .
$$

Since $R$ and $S$ are invertible and Conditions (11e) and (11f) of Definition 4 are satisfied, then $R S^{-1} R-R$ is positive semidefinite and of rank $n_{\text {aw }}$, so there always exists a matrix $N$ satisfying (16). Define the matrix $M \in \mathbb{R}^{n_{\text {aw }} \times n_{\text {aw }}}$ as

$$
\begin{aligned}
& \qquad M:=I+N^{T} R^{-1} N \\
& \text { Finally, } \underset{\text { define }}{Q \in \mathbb{R}^{\left(n_{\mathrm{CL}}+n_{\mathrm{aw}}\right) \times\left(n_{\mathrm{CL}}+n_{\mathrm{aw}}\right)} \text { as }} \text { the } \\
& \qquad Q:=\left[\begin{array}{cc}
R & N \\
N^{T} & M
\end{array}\right]
\end{aligned}
$$

matrix

Step 3) Construct other required matrices.

Construct the matrices $A_{\circ} \in \mathbb{R}^{n \times n}, B_{q \circ} \in$ $\mathbb{R}^{n \times n_{u}}, C_{y \circ} \in \mathbb{R}^{n_{u} \times n}, D_{y q \circ} \in \mathbb{R}^{n_{u} \times n_{u}}, C_{z \circ} \in$ $\mathbb{R}^{n_{z} \times n}, D_{z q \circ} \in \mathbb{R}^{n_{z} \times n_{u}}, H_{1}^{T} \in \mathbb{R}^{n \times\left(n_{\mathrm{aw}}+n_{v}\right)}$, 


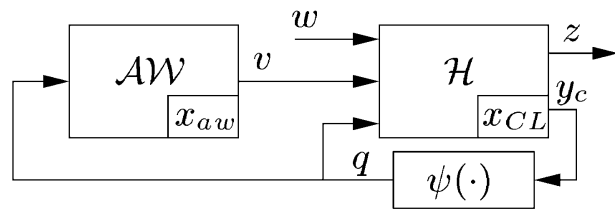

Fig. 5. Equivalent representation of the antiwindup closed-loop system.

$$
\begin{aligned}
& G_{1} \in \mathbb{R}^{\left(n_{\text {aw }}+n_{u}\right) \times n}, G_{2} \in \mathbb{R}^{\left(n_{\text {aw }}+n_{u}\right) \times n_{u}}, H_{2}^{T} \in \\
& \mathbb{R}^{n_{u} \times\left(n_{\text {aw }}+n_{v}\right)}, H_{3}^{T} \in \mathbb{R}^{n_{z} \times\left(n_{\text {aw }}+n_{v}\right)}, B_{w} \in \\
& \mathbb{R}^{n \times n_{w}}, D_{z w} \in \mathbb{R}^{n_{z} \times n_{w}} \text {, and } D_{y w} \in \mathbb{R}^{n_{u} \times n_{w}} \text { as } \\
& \text { follows: } \\
& A_{\circ}=\left[\begin{array}{cc}
A_{\mathrm{CL}} & 0 \\
0 & 0
\end{array}\right] \quad B_{q \circ}=\left[\begin{array}{c}
B_{\mathrm{CL}, q} \\
0
\end{array}\right] \\
& C_{y \circ}=\left[\begin{array}{ll}
C_{\mathrm{CL}, y} & 0
\end{array}\right] \\
& D_{y q \circ}=D_{\mathrm{CL}, y q} \quad C_{z \circ}=\left[\begin{array}{ll}
C_{\mathrm{CL}, z} & 0
\end{array}\right] \quad D_{z q \circ}=D_{\mathrm{CL}, z q} \\
& H_{1}^{T}=\left[\begin{array}{cc}
0 & B_{\mathrm{CL}, v} \\
I_{n_{\mathrm{aw}}} & 0
\end{array}\right] \quad G_{1}=\left[\begin{array}{cc}
0 & I_{n_{\mathrm{aw}}} \\
0 & 0
\end{array}\right] \\
& G_{2}=\left[\begin{array}{c}
0 \\
I_{n_{u}}
\end{array}\right] \\
& H_{2}^{T}=\left[\begin{array}{ll}
0 & D_{\mathrm{CL}, y v}
\end{array}\right] \quad H_{3}^{T}=\left[\begin{array}{ll}
0 & D_{\mathrm{CL}, z v}
\end{array}\right] \\
& B_{w}=\left[\begin{array}{c}
B_{\mathrm{CL}, w} \\
0
\end{array}\right] \quad D_{z w}=D_{\mathrm{CL}, z w} \quad D_{y w}=D_{\mathrm{CL}, y w}
\end{aligned}
$$

\section{Step 4) Construct and solve the antiwindup compensator} LMI.

Stack the matrices of the antiwindup compensator (6) in a single matrix $\Lambda \in \mathbb{R}^{\left(n_{\text {aw }}+n_{v}\right) \times\left(n_{\text {aw }}+n_{u}\right)}$ as follows:

$$
\Lambda:=\left[\begin{array}{cc}
\Lambda_{1} & \Lambda_{2} \\
\Lambda_{3} & \Lambda_{4}
\end{array}\right]
$$

Choose any $\delta \in \mathbb{R}, \delta>0$ and define $U=\delta V_{s}^{-1}$. Based on the matrices determined in Steps 2) and 3) of this procedure, construct the matrices

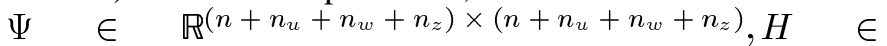
$\mathbb{R}^{\left(n_{\text {aw }}+n_{v}\right) \times\left(n+n_{u}+n_{w}+n_{z}\right)}, \quad$ and $\quad G$ $\mathbb{R}^{\left(n_{\text {aw }}+n_{u}\right) \times\left(n+n_{u}+n_{w}+n_{z}\right)}$ as shown in (21a)-(21c) at the bottom of the page. Finally, compute the matrix $\Lambda$ associated with the desired antiwindup compensator by solving the LMI

$$
\Psi+G^{T} \Lambda^{T} H+H^{T} \Lambda G<0 .
$$

Theorem 4: Given the plant $\mathcal{P}$, the controller $\mathcal{C}$, an integer $n_{\text {aw }}$, a scalar $\tilde{\gamma}$ and a solution $(R, S, \gamma)$ to $\operatorname{MC}\left(\mathcal{P}, \mathcal{C}, n_{\text {aw }}, \tilde{\gamma}\right)$, the LMI (22) constructed according to Procedure 1 is guaranteed to be solvable for $\Lambda$. Furthermore, the solution $\Lambda$ defines the matrices of a linear antiwindup compensator (6) of order $n_{\text {aw }}$ that guarantees well-posedness and quadratic performance of level $\tilde{\gamma}$.

\section{Proof: See Section V.}

Remark 9: To overcome implementation problems, it might be desirable for the antiwindup compensator arising from Procedure 1 to be strictly proper. At least for the case when the controller (2) is strictly proper (namely, $D_{c, y}=0$ and $D_{c, w}=0$ ), this is possible without increasing the performance level $\tilde{\gamma}$ but increasing the dimension of the antiwindup compensator (6) by adding $n_{u}$ states. Indeed, the conditions of Theorem 2 hold for a given $\tilde{\gamma}$ if and only if they hold for some $\bar{\gamma}=\tilde{\gamma}-\delta_{\gamma}$, with $\delta_{\gamma}$ sufficiently small. Then, following a singular perturbation approach (see, e.g., [14, Sec. 9.4]), it can be shown that there exists a sufficiently small constant $\mu>0$ such that the same antiwindup compensator augmented with the filter

$$
\mu \dot{q}=-q+u_{f}
$$

located at its input (namely, choosing $u_{f}=\psi\left(y_{c}\right)$ ) still guarantees well-posedness and quadratic performance of level $\tilde{\gamma}$. Indeed, defining the new state variable $\xi:=q-\psi\left(y_{c}\right)$, a singular perturbation argument allows us to prove a relation similar to (7) for the new antiwindup closed-loop system. In particular, taking any $d \in(0,1)$ a new (Lipschitz) Lyapunov function $\bar{V}(x, \xi):=(1-d) V(x)+d \xi^{2}$ can be shown to satisfy (7) for a smaller $\epsilon$ but the same original value for $\tilde{\gamma}$ (this is possible by the preliminary insertion of the margin $\delta_{\gamma}$ ). 0

Remark 10: When the saturation function is decentralized (consequently, by Remark 5, $U$ can be selected as a diagonal positive definite unknown), the static antiwindup construction in Procedure 1 (with $n_{\text {aw }}=0$ ) corresponds to the optimal static antiwindup construction proposed in [22], where the matrix $U$ is an unknown diagonal positive-definite matrix (therein $U^{-1}$ is referred to as the "stability multiplier") and the parameter $Q=R$, instead of being determined in Step 2), is undetermined and considered as an extra unknown variable in the inequality (22). Indeed, due to the simpler structure of the problem when $n_{\text {aw }}=0$ (causing $G_{1}=0$ ), inequality (22) turns out to be linear in the unknowns $Q, U, \Lambda_{4} U$, and $\gamma$, hence being solvable through a single-step solution, where $\gamma$ can be once again minimized in a convex way. Although the stability multiplier was employed in [22] to improve the antiwindup performance, an interesting implication of Theorem 2 is that since the conditions

$$
\begin{aligned}
\Psi & =\left[\begin{array}{cccc}
Q A_{\circ}^{T}+A_{\circ} Q & B_{q \circ} U+Q C_{y \circ}^{T} & B_{w} & Q C_{z \circ}^{T} \\
U B_{q \circ}^{T}+C_{y \circ} Q & D_{y q \circ} U+U D_{y q \circ}^{T}-2 U & D_{y w} & U D_{z q \circ}^{T} \\
B_{w}^{T} & D_{y w}^{T} & -\gamma I & D_{z w}^{T} \\
C_{z \circ} Q & D_{z q \circ} U & D_{z w} & -\gamma I
\end{array}\right] \\
H & =\left[\begin{array}{llll}
H_{1} & H_{2} & 0 & H_{3}
\end{array}\right] \\
G & =\left[\begin{array}{llll}
G_{1} Q & G_{2} U & 0 & 0
\end{array}\right] .
\end{aligned}
$$


$\operatorname{MC}\left(\mathcal{P}, \mathcal{C}, n_{\mathrm{aw}}, \tilde{\gamma}\right)$ are independent of $V_{s}$, then the minimum achievable performance level does not depend on the stability multiplier.
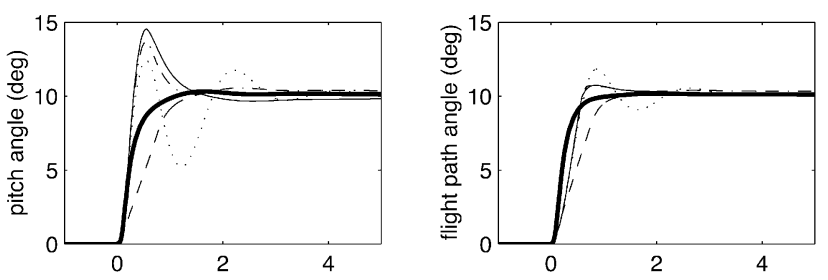

In this section, the antiwindup construction proposed in Section III-C is applied to two linear windup-prone control systems. The first one is a simulation example that illustrates the effectiveness of the construction in the nontrivial case of a multiple-input-multiple-output system. The second one is an experimental application that shows the success of our algorithms when applied to practical control problems. In particular, the application that we have chosen exhibits a difficult windup problem for which static antiwindup is not even capable of guaranteeing quadratic stability (this is verified by checking the conditions in Theorem 3 ) and the more sophisticated plant-order dynamic antiwindup compensation scheme is necessary.

Example 1 (The Longitudinal Dynamics of an F8 Aircraft [13], [19]): Consider a fourth-order linear model of the longitudinal dynamics of the F8 aircraft and the eighth order linear unconstrained controller introduced in [13]. The two inputs to the plant are the elevator angle and the flaperon angle, each one limited between \pm 25 degrees and the two outputs of the plant are the pitch angle and the flight path angle. The controller input is the difference between the plant output and the reference input. The authors of [13] observe a substantial performance loss when the plant input is subject to saturation and propose a reference governor scheme for antiwindup purposes. We will compare their result to the antiwindup compensators designed using the methods in this paper.

The methods in this paper depend on the realization of the unconstrained controller. Using the matrices $\mathbf{A}_{\mathbf{a}}, \mathbf{B}_{\mathbf{a}}, \mathbf{C}_{\mathbf{a}}, \mathbf{H}$, and $\mathbf{G}$ defined in [13], choose the realization of the controller according to

$$
\begin{aligned}
A_{c} & =\left[\begin{array}{cc}
\mathbf{A}_{\mathbf{a}}+\mathbf{B}_{\mathbf{a}} \mathbf{G}-\mathbf{H C}_{\mathbf{a}} & 0 \\
\mathbf{G} & 0
\end{array}\right] \quad-B_{c, w}=B_{c, y}=\left[\begin{array}{c}
\mathbf{H} \\
0
\end{array}\right] \\
C_{c} & =\left[\begin{array}{ll}
0 & I
\end{array}\right]
\end{aligned}
$$
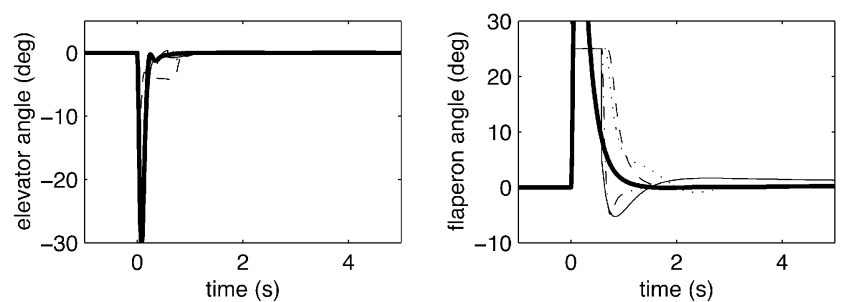

Fig. 6. Example 1. Comparison of the unconstrained response (bold solid) and of the saturated response (dotted) to the static (dash-dotted) and dynamic (thin solid) antiwindup designs with $z=y-w$ and to the scheme of Kapasouris $e t$ al. (dashed).

discussed thus far, are shown in Fig. 6, where the bold solid line is the unconstrained trajectory, the dotted line is the saturated trajectory, the dashed line is the antiwindup response with the method of [13], the dash-dotted line is our static antiwindup response, and the thin solid line is our plant-order antiwindup response. Both of the antiwindup closed-loop system responses have significant overshoot and are, perhaps, undesirable.

Next, we will show that the antiwindup trajectories can be significantly improved by selecting a different performance output. We observe the most substantial degradation in performance of the saturated closed-loop trajectories is the large overshoot and settling time of the pitch angle. For this reason, we select the performance objective to be composed of the pitch angle error and the angular acceleration due to the plant state on the pitch angle. In particular, we will define the performance output via the matrices

$$
\begin{aligned}
C_{p, z} & =\left[\begin{array}{cccc}
0 & 0 & 0 & \frac{3}{4} \\
-.8 & -.0006 & -12 & 0
\end{array}\right] \\
D_{p, z u} & =0_{2 \times 2} \\
D_{p, z w} & =\left[\begin{array}{cc}
-\frac{3}{4} & 0 \\
0 & 0
\end{array}\right] .
\end{aligned}
$$

and $D_{c, y}$ and $D_{c, w}$ are zero matrices of appropriate dimensions. By selecting the performance output $z=y-w$ where $w$ denotes the reference input, a static antiwindup compensator can be constructed using Procedure 1 with $n_{\mathrm{aw}}=0$ which guarantees performance level $\gamma=22.19$ and the resulting antiwindup compensator consists of the gain is as shown in the equation at the bottom of the page. Similarly, a plant-order antiwindup compensator can be constructed using the same performance output and Procedure 1 with $n_{\text {aw }}=n_{p}$, resulting in an antiwindup compensator with guaranteed performance level $\gamma=19.39$. To save space, the constructed matrices are not written here. The antiwindup closed-loop system response, and the other responses
A static antiwindup compensator can now be constructed using Procedure 1 with $n_{\text {aw }}=0$ which guarantees performance level $\gamma=26.18$ and the resulting antiwindup compensator consists of the gain as shown in the the equation at the bottom of the page. Similarly, a plant-order antiwindup compensator can be constructed using the same pitch angle performance output and Procedure 1 with $n_{\text {aw }}=n_{p}$, resulting in an antiwindup compensator with guaranteed performance level $\gamma=22.91$. To save space, the constructed matrices are not written here. The antiwindup closed-loop system response, and some of the other

$$
\Lambda_{4}=\left[\begin{array}{cccccccccc}
6.1077 & 10.113 & -5.1947 & -1267 & -0.17647 & 0.89373 & 6.2456 & 11.053 & -0.90667 & 1.5318 \\
-1.9882 & 9.6373 & -3.8543 & -566.8 & -0.24158 & 0.31719 & -1.9261 & 10.102 & 0.05948 & 0.01987
\end{array}\right]^{T} .
$$



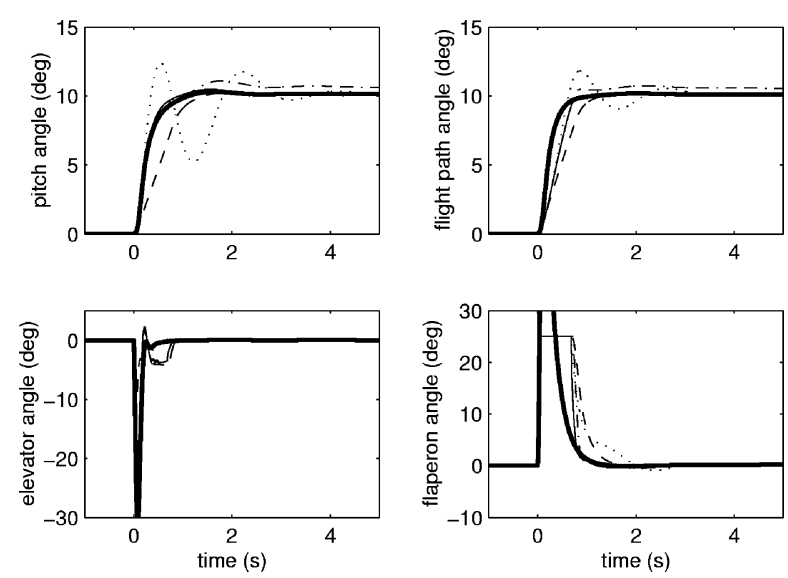

Fig. 7. Example 1. Comparison of the unconstrained response (bold solid) and of the saturated response (dotted) to the static (dash-dotted) and dynamic (thin solid) antiwindup designs with pitch angle performance output and to the scheme of Kapasouris et al. (dashed).

responses discussed previously, are shown in Fig. 7 where the bold solid line is the unconstrained trajectory, the dotted line is the saturated trajectory, the dashed line is the antiwindup response with the method of [13], the dash-dotted line is our static antiwindup response, and the thin solid line is our plantorder antiwindup response. The trajectories of this antiwindup closed-loop system designed using the pitch angle performance output, particularly with plant-order antiwindup, are highly desirable and are a marked improvement over the scheme proposed in [13].

Example 2 (An Experimental Example): The cart-springpendulum system shown in Figs. 8 and 9 (which is available at the Control and Computation Laboratory at the University of California, Santa Barbara) consists of a cart restricted to motion on a straight and level track which is attached via a spring to a fixed wall. A pendulum is suspended from the cart by a hinge so as to be constrained to the vertical plane defined by the track. The cart is equipped with a DC motor that exerts a torque to a small toothed wheel which, in turn, applies a force on the cart. The system will be disturbed by a sharp tap on the pendulum that comes from a human hand. For the purpose of deriving a model, the experimental system will be considered to be composed of a massless spring attached to a frictionless cart from which a slender rod freely hangs.

The output of the system is the position $p$ of the cart, in meters, relative to the spring's equilibrium point and the angular position $\theta$ of the pendulum, in radians, relative to the vertical; both positions are measured with optical encoders. The physical inputs of the system are the voltage $u$ applied to the armature of the dc motor, in Volts, and a disturbance force $w$, in Newtons. The force from the motor $f$, in Newtons, is modeled as $f=k_{1} u-k_{2} \dot{p}$. The operating range of the control input is constrained by the range of the D/A converter, $[-5,5]$ Volts (which, incidentally, nearly covers the entire operating range of

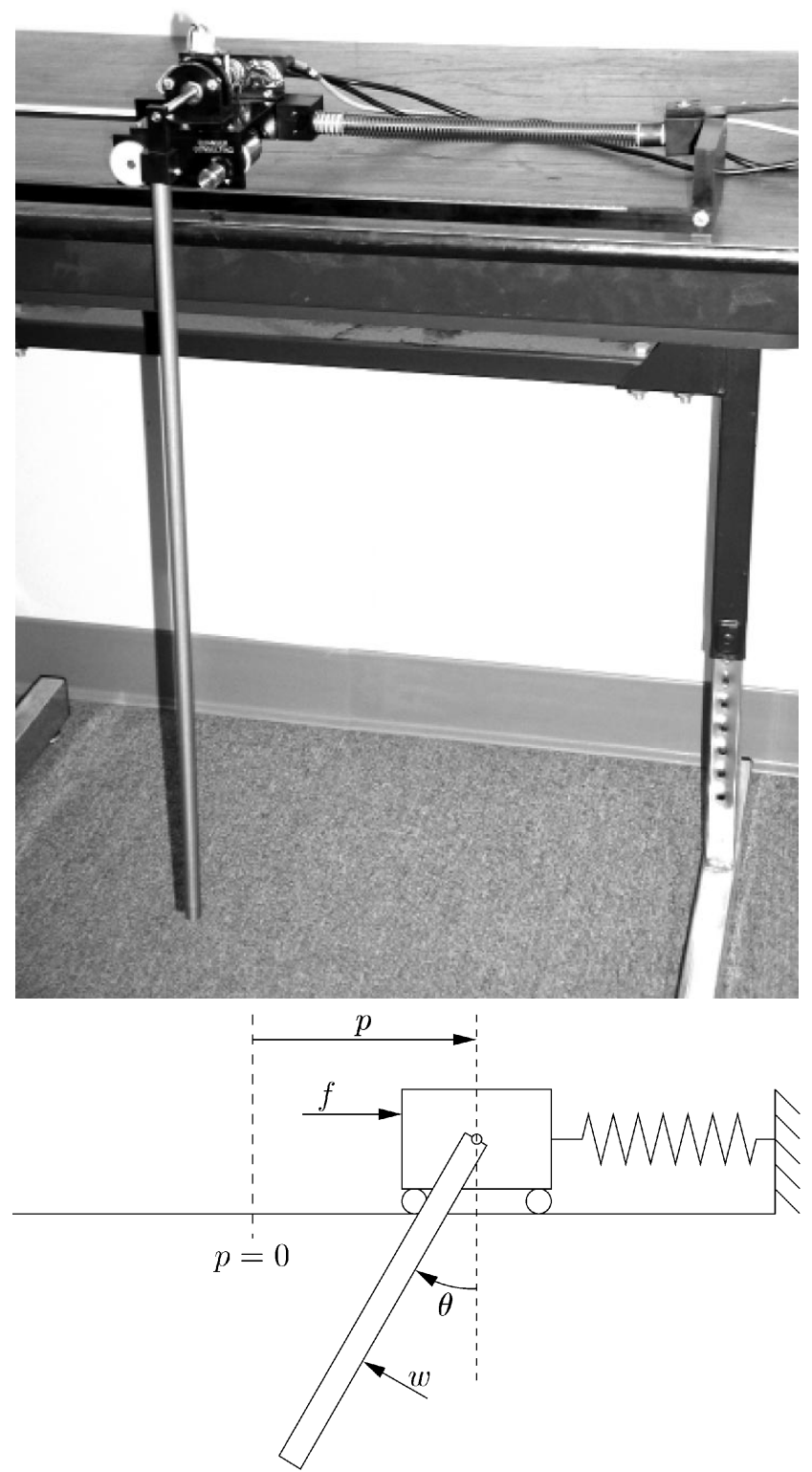

Fig. 8. Damped mass-spring-pendulum system in Example 2.

the DC motor, $[-6,6]$ Volts). The disturbance $w$ is a force in the plane of motion orthogonal to the pendulum of length $2 l$ and acts at a distance of $(4 / 3) l$ from the cart-pendulum hinge. A nonlinear model of the system can be derived by applying standard Euler-Lagrange techniques. Moreover, defining the plant

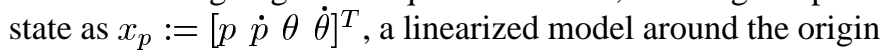
is given by (1) and

$$
A_{p}=\left[\begin{array}{cccc}
0 & 1 & 0 & 0 \\
-330.46 & -12.15 & -2.44 & 0 \\
0 & 0 & 0 & 1 \\
-812.61 & -29.87 & -30.10 & 0
\end{array}\right]
$$
$\Lambda_{4}=$ $\begin{array}{rrr}14.339 & -55.258 & -0.10926\end{array}$
528.52
2.4325
-7.6946
5.9347
0.01282
0.54967
0.30214
14.406
$-55.168$
$-0.97438$
$0.69361-76.628$
527.19
$-0.13474$
$\left.\begin{array}{c}-0.019895 \\ -0.76157\end{array}\right]^{T}$ 

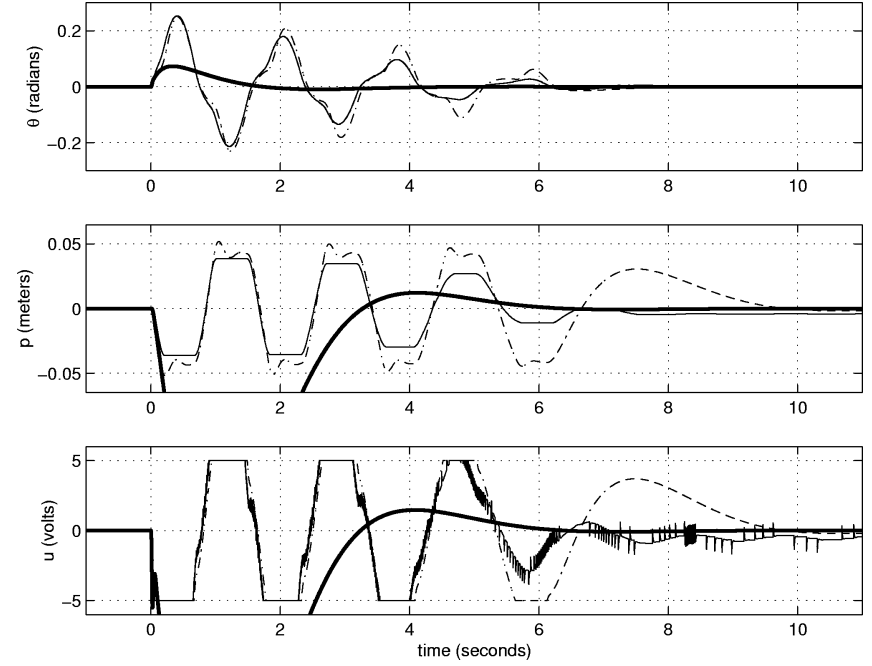

Fig. 9. Example 2. Response to the larger pendulum tap. Simulated unconstrained response (bold solid); simulated saturated response (dash-dotted); experimental saturated response (thin solid).

$$
\begin{aligned}
& B_{p, u}=\left[\begin{array}{c}
0 \\
2.71762 \\
0 \\
6.68268
\end{array}\right] \quad B_{p, w}=\left[\begin{array}{c}
0 \\
0 \\
0 \\
15.61
\end{array}\right] \\
& C_{p, y}=\left[\begin{array}{llll}
1 & 0 & 0 & 0 \\
0 & 0 & 1 & 0
\end{array}\right] \quad D_{p, y u}=\left[\begin{array}{l}
0 \\
0
\end{array}\right] \\
& D_{p, y w}=\left[\begin{array}{l}
0 \\
0
\end{array}\right]
\end{aligned}
$$

where $A_{p}$ is Hurwitz.

Suppose the system is allowed to come to rest before it is disturbed and we are interested in the response of the system due to two test pendulum taps, one small ${ }^{3}$ and one five times larger. Suppose further the objective is to return the pendulum and cart quickly and gently to their equilibrium after the smaller taps and gracefully handle the larger taps to the pendulum. Following an LQG construction, an observer based controller of the form (2) is designed where

$$
\begin{aligned}
K & =\left[\begin{array}{llll}
64.81 & 213.12 & 1242.27 & 85.82
\end{array}\right] \\
L & =\left[\begin{array}{cccc}
64 & 2054 & -8 & -1432 \\
-8 & -280 & 142 & 10169
\end{array}\right]^{T}
\end{aligned}
$$

$A_{c}:=A_{p}-B_{p, u} K-L C_{p, y}, B_{c, y}:=L, C_{c}:=-K$, and $B_{c, w}, D_{c, y}$, and $D_{c, w}$ are zero matrices of appropriate dimensions.

For the simulations reported here, we have used the linearized model of the plant. Indeed, the resulting trajectories are almost the same as the corresponding ones with the nonlinear Euler-Lagrange model, thus confirming the appropriateness of the linear approximation for our operating conditions. For the smaller pendulum tap, the plant input does not saturate and the unconstrained response is deemed desirable, both in simulation and in experiment. The settling time for the pendulum is approximately $1.5 \mathrm{~s}$ and for the cart, it is $3 \mathrm{~s}$. The larger pendulum

${ }^{3}$ For simulation purposes, the smaller pendulum tap is modeled a constant force of 1.588 Newton with duration $0.01 \mathrm{~s}$. taps, however, give rise to undesirable closed-loop behavior, i.e., the settling time is severely deteriorated. In Fig. 9, the bold solid curve represents the simulated (ideal) unconstrained response, the dash-dotted curve represents the simulation of the saturated response and the thin solid curve represents the corresponding experiment. ${ }^{4}$ The noticeable mismatch between the thin solid and the dash-dotted curves is cause by unmodeled effects of the experimental device: mainly backlash and stiction affecting the movement of the cart on the track. Besides these unmodeled phenomena (which cause significant differences, especially on the tails of the responses), the fourth order model represents sufficiently well the dynamics of our experimental system.

Based on the antiwindup construction proposed in Procedure 1, the undesired behavior of Fig. 9 can be mitigated by augmenting the experimental control system according to the diagram in Fig. 3. To determine an optimal selection of the antiwindup compensator matrices we first choose a performance output $z$. By inspecting Fig. 9, we see that for the larger pendulum taps, the pendulum swings wildly causing the cart to chase after the pendulum, almost in vain. To reduce quickly the magnitude of $\theta$, we choose the matrices related to the performance output $z$ as follows: $C_{p, z}=\left[\begin{array}{llll}0 & 0 & 1 & 0\end{array}\right], D_{p, z u}=0, D_{p, z w}=0$. A first antiwindup design attempt is carried out by selecting $k=0$ to explore feasibility of static antiwindup compensation. Unfortunately, for this system, the associated LMIs (12) in Proposition 1 are unfeasible. ${ }^{5}$ As a further step, we move to dynamic antiwindup compensation of order $k=n_{p}$, which, based on the asymptotic stability of the plant, is guaranteed to be feasible by Theorem 3. To construct this compensator, Procedure 1 is applied with $n_{\mathrm{aw}}=n_{p}$ and the following compensation matrices are obtained, which guarantee a performance level of $\gamma=181.82$ :

$$
\begin{aligned}
& \Lambda_{1}=\left[\begin{array}{cccc}
-65.02 & 198.43 & 98.11 & -66.75 \\
223.94 & -697.09 & -347.39 & 247.24 \\
41.17 & -98.10 & -47.56 & 55.25 \\
-121.39 & 309.97 & 138.31 & -131.52
\end{array}\right] \\
& \Lambda_{2}=\left[\begin{array}{c}
0.0688 \\
-0.2620 \\
-0.0637 \\
0.1559
\end{array}\right] \\
& \Lambda_{3}=\left[\begin{array}{cccc}
41.22 & -160.42 & -106.41 & 82.03 \\
-3469.09 & 8318.57 & 3423.87 & -2388.49 \\
-162.51 & 386.26 & -35.56 & 71.07 \\
-4584.37 & 9490.06 & -11350.16 & 11407.08 \\
587.11 & -1687.16 & -821.25 & 632.86
\end{array}\right] \\
& \Lambda_{4}=\left[\begin{array}{c}
-0.0622 \\
2.9070 \\
0.2338 \\
5.5623 \\
0
\end{array}\right]
\end{aligned}
$$

\footnotetext{
${ }^{4}$ Although a continuous time controller has been designed, it is implemented in discrete time. We allow Quanser Consulting Inc. software, WinCon 3.1, to convert our continuous time controller to discrete time using the Runge-Kutta fixed-step solver with sampling time $0.0005 \mathrm{~s}$.

${ }^{5}$ Unfeasibility was determined due the inability of the MATLAB LMI Control Toolbox to find a feasible solution to the LMIs (12).
} 

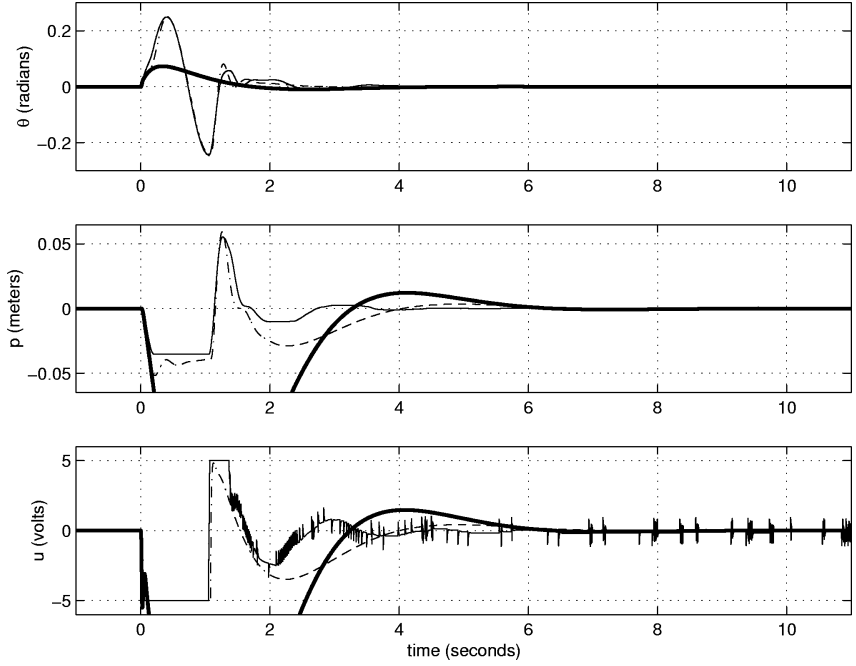

Fig. 10. Example 2. Response to the larger pendulum tap. Simulated unconstrained response (bold solid); simulated response with antiwindup (dash-dotted); experimental response with antiwindup (thin solid).

The thin solid curve in Fig. 10 represents the experimental response of the closed-loop system with dynamic antiwindup compensation to the same disturbance that generates the undesirable response represented in Fig. 9. Similarly to Fig. 9, the dash-dotted curve represents a simulation of the closed-loop with the linear plant model, while the bold solid curve represents a simulation of the unconstrained closed-loop system's response. A comparison between the thin solid responses in Figs. 10 and 9 illustrates that the insertion of the antiwindup compensator greatly improves the experimental response to the larger pendulum taps, while structurally preserving the desirable performance of the (previously designed) unconstrained controller for the smaller pendulum taps. It should be recognized that the tails of the simulated responses are quite different from the experimental ones because of the unmodeled effects commented above. Nevertheless, the plant model is mostly accurate in the operating conditions where the plant input is close to the saturation limits. These are the operating conditions of interest for the antiwindup action, hence a more accurate model of the plant does not seem to be necessary for the antiwindup design.

\section{Proof of the MaIN Result}

\section{A. Proof of Theorem 1}

To prove Theorem 1, the following lemmas will be useful. The proofs of Lemmas 2 and 3 can be carried as in [33].

Lemma 2: Consider a locally Lipschitz function $F: \mathbb{R}^{n} \mapsto$ $\mathbb{R}^{n}$ and assume that the Jacobian of $F$ satisfies

$$
J F(x) \in \mathcal{M}, \quad \text { for almost all } x \in \mathbb{R}^{n}
$$

where the set $\mathcal{M}$ is compact, convex, and each matrix in $\mathcal{M}$ is nonsingular. Then there exists a (unique) globally Lipschitz function $G: \mathbb{R}^{n} \mapsto \mathbb{R}^{n}$ such that $F(G(x))=x$ for all $x \in \mathbb{R}^{n}$. Equivalently, $F$ is a homeomorphism with globally Lipschitz inverse.

Lemma 3: Given two square matrices $D$ and $V=V^{T}>0$, if $-2 V+V D+D^{T} V<0$ then $I-D \Delta$ is nonsingular for all $\Delta$ such that the linear map $z \mapsto \Delta z$ belongs to the sector $[0, I]_{V}$.

Lemma 4: Given any symmetric positive-definite matrix $V_{s}$, the function $\phi(\cdot)$ belongs to $\Phi_{V_{s}}$ if and only if the function $\psi\left(y_{c}\right):=y_{c}-\phi\left(y_{c}\right)$ belongs to $\Phi_{V_{s}}$.

Proof (Sufficiency): Assume $\phi(\cdot)$ belongs to $\Phi_{V_{s}}$. Clearly, $\psi(\cdot)$ is globally Lipschitz. Moreover, since $\langle\phi(y)-y, \phi(y)\rangle_{V_{s}} \leq 0$ for all $y$, then $\langle-\psi(y), y-\psi(y)\rangle_{V_{s}} \leq 0$ for all $y$, namely $\psi(\cdot)$ belongs to sector $[0, I]_{V_{s}}$. Moreover, since $J \psi\left(y_{c}\right)=I-J \phi\left(y_{c}\right)$ whenever $J \phi\left(y_{c}\right)$ exists it follows that $\langle J \psi(y), I-J \psi(y)\rangle_{V_{s}} \geq 0$ for almost all $y \in \mathbb{R}^{n_{u}}$. Thus $\psi(\cdot)$ belongs to $\Phi_{V_{s}}$. The necessity can be proven by swapping the functions $\phi(\cdot)$ and $\psi(\cdot)$ in the previous proof.

The following facts will also be useful for the proof of Theorem 1.

Fact 1: By noting that $y_{c}$ and $z$ are linear functions of $x, q$, and $w$, writing the upcoming (23) in matrix inequality form and taking its Schur complement [4, p. 7], it can be shown that given $P=P^{T}>0$, and $V=x^{T} P x$, where its derivative along the dynamics of the system (8), (9) is $\dot{V}=2 x^{T} P\left(A x+B_{q} q+\right.$ $\left.B_{w} w\right)$, then

$$
\begin{aligned}
\dot{V}+\frac{1}{\gamma} z^{T} z-\gamma w^{T} w+2 \tau q^{T} W\left(y_{c}-q\right) & <0 \\
\forall(x, q, w) & \neq 0
\end{aligned}
$$

if and only if the equation shown at the bottom of the page holds.

Fact 2: By employing the $\mathcal{S}$-procedure [4, p. 24], it is shown that given any symmetric positive definite matrix $W$ and (as in Fact 1) $\dot{V}=2 x^{T} P\left(A x+B_{q} q+B_{w} w\right)$, if

1) there exists a scalar $\tau \geq 0$ such that

$\dot{V}+\frac{1}{\gamma} z^{T} z-\gamma w^{T} w+2 \tau q^{T} W\left(y_{c}-q\right)<0 \quad \forall(x, q, w) \neq 0$

then

2)

$$
\dot{V}+\frac{1}{\gamma} z^{T} z-\gamma w^{T} w<0
$$

for all $(x, q, w) \neq 0$ such that

$$
q^{T} W\left(y_{c}-q\right)=q^{T} W\left(C_{y} x+D_{y q} q+D_{y w} w-q\right) \geq 0 .
$$

$$
\left[\begin{array}{cccc}
A^{T} P+P A & P B_{q}+\tau C_{y}^{T} W & P B_{w} & C_{z}^{T} \\
B_{q}^{T} P+\tau W C_{y} & \tau\left(W D_{y q}+D_{y q}^{T} W-2 W\right) & \tau W D_{y w} & D_{z q}^{T} \\
B_{w}^{T} P & \tau D_{y w}^{T} W & -\gamma I & D_{z w}^{T} \\
C_{z} & D_{z q} & D_{z w} & -\gamma I
\end{array}\right]<0 .
$$


In addition, if there exists at least one selection $\left(x^{*}, q^{*}, w^{*}\right)$ such that

$$
q^{* T} W\left(C_{y} x^{*}+D_{y q} q^{*}+D_{y w} w^{*}-q^{*}\right)>0
$$

then item 2) implies item 1). (26).

Fact 3: There exists a selection $\left(x^{\star}, q^{\star}, w^{\star}\right)$ that satisfies

Proof: If there exist $x^{\star}, w^{\star}$ such that $\left[C_{y} x^{\star}+D_{y w} w^{\star}\right] \neq$ 0 , then pick $q^{\star}=\varepsilon\left[C_{y} x^{\star}+D_{y w} w^{\star}\right]$ with $\varepsilon$ sufficiently small to satisfy (26). Conversely, if $C_{y} x+D_{y w} w=0$ for all $(x, w)$, then the controller $\mathcal{C}$ is identically zero. In this trivial case, $u=0$ for all times. Namely, since the saturation never activates, the antiwindup problem is nonexistent. From a more system theoretic viewpoint, in this case the optimal performance $\gamma$ is the $\mathcal{L}_{2}$ gain of the open-loop plant, and an antiwindup compensator that achieves this performance level is the identically zero antiwindup compensator.

\section{Proof of Theorem 1:}

Necessity Assume that for a given plant, controller and antiwindup compensator of order $n_{\text {aw }}$, well-posedness and quadratic performance of level $\tilde{\gamma}$ are guaranteed in the sense of Definition 3. Lemma 4 guarantees $\psi(\cdot)$ belongs to $\Phi_{V_{s}}$, and therefore $\left\langle q, y_{c}-q\right\rangle_{V_{s}} \geq 0$. Hence, by inequality (7), there exists a quadratic Lyapunov function $V(x)=x^{T} P x$ where $P=P^{T}>0$ such that item 2 in Fact 2 is satisfied with $W=V_{s}$ and $\gamma=\tilde{\gamma}$. Combined with Fact 3, Fact 2 implies that there exists a constant $\tau \geq 0$ that satisfies (24). Finally, by Fact 1, (27), shown at the bottom of the page, holds. Moreover, since all block diagonal terms in (27) must be nonzero, then $\tau \neq 0$. Defining $Q:=P^{-1}$ and $U:=\tau^{-1} V_{s}^{-1}$ and then premultiplying and postmultiplying (27) by the symmetric block diagonal matrix $\operatorname{diag}(Q, U, I, I)$, it follows that there exists $Q=Q^{T}>0$ and $\delta:=\tau^{-1}>0$ that satisfy (10a), as desired.

Sufficiency If there exist $Q, \gamma$, and $\delta>0$ that satisfy (10), define $P:=Q^{-1}$ and $\tau:=\delta^{-1}$ and premultiply and postmultiply (10a) by the symmetric block diagonal matrix $\operatorname{diag}\left(P, \tau V_{s}, I, I\right)$. The resulting inequality guarantees (27) because $\tilde{\gamma} \geq \gamma$. Then, Fact 1 and Fact 2 guarantee that the function $V(x)=x^{T} P x$ satisfies item 2 in Fact 2 with $W=V_{s}$. Since $q=\psi\left(y_{c}\right)$ and $\psi(\cdot)$ belongs to $[0, I]_{V_{s}}$, inequality (25b) is always satisfied by the trajectories of the closed-loop system (1), (2), (5), (6). Hence, since the inequality in (25a) is strict, there exists a small enough $\epsilon>0$ such that inequality (7) in item 2 of Definition 3 is guaranteed.

To show well-posedness in item 1) of Definition 3, rewrite the interconnection of (8) and the middle equation of (9) as

$$
H\left(y_{c}\right):=y_{c}-D_{y q} \psi\left(y_{c}\right)=C_{y} x+D_{y w} w
$$

where $H(\cdot)$ is globally Lipschitz. Since, by Lemma 4, the function $\psi(\cdot)$ belongs to $\Phi_{V_{s}}$, then almost everywhere, $\Delta=J \psi\left(y_{c}\right)$ is such that $\langle\Delta, I-\Delta\rangle_{V_{s}} \geq 0$. This can be rewritten as

$$
-2 \Delta^{T} V_{s} \Delta+V_{s} \Delta+\Delta^{T} V_{s} \geq 0
$$

where $\Delta=J \psi\left(y_{c}\right)$ almost everywhere. Then, for almost all $y_{c}$, the Jacobian of $H\left(y_{c}\right)$ satisfies

$$
\begin{aligned}
J H\left(y_{c}\right) \in\left\{\left(I-D_{y q} \Delta\right), \Delta:-\right. & 2 \Delta^{T} V_{s} \Delta \\
& \left.+V_{s} \Delta+\Delta^{T} V_{s} \geq 0\right\}=: J \mathcal{H}
\end{aligned}
$$

where the set $J \mathcal{H}$ is compact by the boundedness of $\Delta$ and because the inequality in (28) is nonstrict. The set $J \mathcal{H}$ is also convex because, by Schur complement, inequality (28) can be written as an LMI in $\Delta$. Furthermore, since the diagonal entries of (10a) are negative definite, then $-2 V_{s}+V_{s} D+D^{T} V_{s}<0$ and, by Lemma 3, each matrix in the set $J \mathcal{H}$ is nonsingular. Then, by Lemma 2 there exists a (unique) globally Lipschitz function $\zeta(\cdot)$ such that $y_{c}=\zeta\left(C_{y} x+D_{y w} w\right)$. Finally, the Lipschitz property of the right-hand side of (9) guarantees the existence and uniqueness of solutions, thus proving well-posedness of the interconnection between (8) and (9).

\section{B. Proof of Theorems 2 and 4}

A key step in the proof of Theorems 2 and 4 is the connection between the matrix conditions $\operatorname{MC}\left(\mathcal{P}, \mathcal{C}, n_{\text {aw }}, \tilde{\gamma}\right)$ in Definition 4, the LMIs for analysis (10) in Theorem 1, and the LMI (22) in the final step of Procedure 1. The LMIs (10a) and (22) coincide but are in different unknowns; the LMI (10a) is in the unknown $Q$ and the LMI (22) is in the unknown $\Lambda$. Indeed, since the system (9) represented by the diagram in Fig. 4 coincides with the system (6), (15) represented in Fig. 5, the matrices in (9) can be expressed in terms of the matrices in (6), (15). Within this equivalence, it is easy to check that the matrices $B_{w}, D_{y w}$, and $D_{z w}$ in (9) coincide with those defined in (19c) and the remaining matrices in (9) satisfy

$$
\begin{aligned}
A & =A_{\circ}+H_{1}^{T} \Lambda G_{1} \quad C_{y}=C_{y \circ}+H_{2}^{T} \Lambda G_{1} \\
C_{z} & =C_{z \circ}+H_{3}^{T} \Lambda G_{1} \\
B_{q} & =B_{q \circ}+H_{1}^{T} \Lambda G_{2} \quad D_{y q}=D_{y q \circ}+H_{2}^{T} \Lambda G_{2} \\
D_{z q} & =D_{z q \circ}+H_{3}^{T} \Lambda G_{2} .
\end{aligned}
$$

The following theorem establishes the equivalence between the feasibility of the matrix conditions $\operatorname{MC}\left(\mathcal{P}, \mathcal{C}, n_{\mathrm{aw}}, \tilde{\gamma}\right)$ in Definition 4 and the feasibility of the matrix constraints (10) and (22).

Theorem 5:

1) Given the plant $\mathcal{P}$ in (1), controller $\mathcal{C}$ in (2), integer $n_{\text {aw }} \geq$ 0 and scalar $\tilde{\gamma}$, there exist matrices $Q, \Lambda$ and scalars $\gamma, \delta$

$$
\left[\begin{array}{cccc}
A^{T} P+P A & P B_{q}+\tau C_{y}^{T} V_{s} & P B_{w} & C_{z}^{T} \\
B_{q}^{T} P+\tau V_{s} C_{y} & \tau\left(-2 V_{s}+V_{s} D_{y q}+D_{y q}^{T} V_{s}\right) & \tau V_{s} D_{y w} & D_{z q}^{T} \\
B_{w}^{T} P & \tau D_{y w}^{T} V_{s} & -\tilde{\gamma} I & D_{z w}^{T} \\
C_{z} & D_{z q} & D_{z w} & -\tilde{\gamma} I
\end{array}\right]<0 .
$$


satisfying (10) (with the definitions (19), (29)) if and only if the matrix conditions $\operatorname{MC}\left(\mathcal{P}, \mathcal{C}, n_{\text {aw }}, \tilde{\gamma}\right)$ are feasible.

2) Given a feasible solution $(R, S, \gamma)$ to $\operatorname{MC}\left(\mathcal{P}, \mathcal{C}, n_{\text {aw }}, \tilde{\gamma}\right)$, the matrix $Q$ constructed in (16), (17), (18) guarantees that the LMI (22) in the unknowns $(\Lambda, \delta, \gamma)$ is solvable and the arising solution $(Q, \Lambda, \delta, \gamma)$ also satisfies (10) [with the definitions (19) and (29)].

Proof: See Section V-B.

Proof of Theorem 2: The composition of Theorem 1 and item 1 in Theorem 5 imply Theorem 2.

Proof of Theorem 4: Step 1) of Procedure 1 is assumed to be solvable. Steps 2) and 3) are constructive. For Step 4), the matrices (21) can always be constructed based on the matrices computed at the preceding steps. Moreover, by item 2) in Theorem 5, the matrix $Q$ constructed in Step 2 guarantees that the LMI (22) is solvable for $\Lambda$ and any feasible solution $(\Lambda, \delta, \gamma)$ to the LMI (22) is such that $(Q, \Lambda, \delta, \gamma)$ satisfies (10). Hence, by Theorem 1, the antiwindup closed-loop system (8), (9) corresponding to $\Lambda$ is well-posed and guarantees quadratic performance of level $\tilde{\gamma}$.

The following lemmas, proven in [8], [12] and [23], respectively, will be useful for the proof of Theorem 5 .

Lemma 5 (Projection Lemma [8, Lemma 3.1]): Given a symmetric matrix $\Psi \in \mathbb{R}^{m \times m}$ and two matrices $G, H$ of column dimension $m$, consider the problem of finding some matrix $\Lambda$ of compatible dimensions such that

$$
\Psi+G^{T} \Lambda^{T} H+H^{T} \Lambda G<0 .
$$

Denote by $W_{G}, W_{H}$ any matrices whose columns form bases of the null space of $G$ and $H$, respectively. Then (30) is solvable for $\Lambda$ if and only if

$$
\begin{aligned}
& W_{H}^{T} \Psi W_{H}<0 \\
& W_{G}^{T} \Psi W_{G}<0 .
\end{aligned}
$$

Lemma 6 [23]: Let $R, Z \in \mathbb{R}^{n \times n}$ be symmetric positive definite matrices. Then the two conditions

$$
\begin{aligned}
Z-R^{-1} & \geq 0 \\
\operatorname{rank}\left[Z-R^{-1}\right] & \leq n_{\text {aw }}
\end{aligned}
$$

hold if and only if there exist $N \in \mathbb{R}^{n \times n_{\text {aw }}}$ and $M \in \mathbb{R}^{n_{\text {aw }} \times n_{\text {aw }} \text {, }}$ with $M=M^{T}>0$ such that

$$
\left[\begin{array}{cc}
R & N \\
N^{T} & M
\end{array}\right]>0 \quad\left[\begin{array}{cc}
R & N \\
N^{T} & M
\end{array}\right]^{-1}=\left[\begin{array}{ll}
Z & ? \\
? & ?
\end{array}\right]
$$

where? denotes matrix entries that we do not care about.

Proof of Theorem 5: We first prove the necessity part of item 1. According to the definitions (19), (20), (21), and (29), (10a) coincides with (22) as shown in (33) at the bottom of the page. We will apply Lemma 5 to inequality (33) [which coincides with (10a)] to show that there exists a feasible solution $(Q, \Lambda, \gamma, \delta)$ to (10) if and only if the conditions $\operatorname{MC}\left(\mathcal{P}, \mathcal{C}, n_{\text {aw }}, \tilde{\gamma}\right)$ in Definition 1 are feasible. In particular, we will show that (31a) is equivalent to (11a) and that (31b) is equivalent to (11b), that the coupling between (11a) and (11b) through $\Psi$ can be rewritten as (11e), (11f).

Condition (11a): According to (19b), (21b) and the explicit expressions for the matrices in (15), $H$ can be written as (34), shown at the bottom of the page, where $\Delta_{y p}:=\left(I-D_{p, y u} D_{c, y}\right)^{-1}$ and $\Delta_{y c}:=\left(I-D_{c, y} D_{p, y u}\right)^{-1}$ are well defined (namely the matrices in parentheses are invertible) by the well-posedness of the unconstrained interconnection. According to this special structure, a matrix that spans the null space of $H$ is

$$
W_{H}=\left[\begin{array}{cccccc}
I_{n_{p}} & 0 & 0 & -B_{p, u} & 0 & 0 \\
0 & 0 & 0 & 0 & I_{n_{w}} & 0 \\
0 & 0 & 0 & -D_{p, z u} & 0 & I_{n_{z}}
\end{array}\right]^{T} .
$$

Indeed, by the assumption of well-posedness of the unconstrained closed-loop system, $\Delta_{y c}$ is full rank, hence, according to the (34), the dimension of the null space of $H$ is necessarily $n_{p}+n_{z}+n_{w}$. Moreover, the rank of $W_{H}$ is $n_{p}+n_{z}+n_{w}$ and it can be verified by computation that $H W_{H}=0$.

$$
\begin{aligned}
& {\left[\begin{array}{cccc}
Q A^{T}+A Q & B_{q} U+Q C_{y}^{T} & B_{w} & Q C_{z}^{T} \\
U B_{q}^{T}+C_{y} Q & D_{y q} U+U D_{y q}^{T}-2 U & D_{y w} & U D_{z q}^{T} \\
B_{w}^{T} & D_{y w}^{T} & -\gamma I & D_{z w}^{T} \\
C_{z} Q & D_{z q} U & D_{z w} & -\gamma I
\end{array}\right]} \\
& =\Psi+\left[\begin{array}{cccc}
H_{1}^{T} \Lambda G_{1} Q+Q G_{1}^{T} \Lambda^{T} H_{1} & H_{1}^{T} \Lambda G_{2} U+Q G_{1}^{T} \Lambda^{T} H_{2} & 0 & Q G_{1}^{T} \Lambda^{T} H_{3} \\
H_{2}^{T} \Lambda G_{1} Q+U G_{2}^{T} \Lambda^{T} H_{1} & H_{2}^{T} \Lambda G_{2} U+U G_{2}^{T} \Lambda^{T} H_{2} & 0 & U G_{2}^{T} \Lambda^{T} H_{3} \\
0 & 0 & 0 & 0 \\
H_{3}^{T} \Lambda G_{1} Q & H_{3}^{T} \Lambda G_{2} U & 0 & 0
\end{array}\right] \\
& =\Psi+H^{T} \Lambda G+G^{T} \Lambda^{T} H \leq 0 .
\end{aligned}
$$

$$
H=\left[\begin{array}{cccccc}
0 & 0 & I_{n_{\text {aw }}} & 0 & 0 & 0 \\
0 & I_{n_{c}} & 0 & 0 & 0 & 0 \\
\Delta_{y c}^{T} B_{p, u}^{T} & D_{p, y u}^{T} \Delta_{y p}^{T} B_{c, y}^{T} & 0 & \Delta_{y c}^{T} & 0 & \Delta_{y c}^{T} D_{p, z u}^{T}
\end{array}\right]
$$


Assume that, according to (18), the matrix $Q$ is partitioned as

$$
Q=\left[\begin{array}{cc}
R & N \\
N^{T} & M
\end{array}\right]
$$

where

$$
R=\left[\begin{array}{ll}
R_{11} & R_{12} \\
R_{12}^{T} & R_{22}
\end{array}\right]
$$

Then, inequality (31a) can be computed explicitly based on (35) and (21a) with (19a), (19c). After some computations it follows that $W_{H}^{T} \Psi W_{H}<0$ coincides with the inequality in (11a), as desired.

Condition (11b): According to (21c), the matrix $G$ can be factored as follows:

$$
\begin{aligned}
G=G_{O} \bar{T} & =\left[\begin{array}{llll}
G_{1} Q & G_{2} U & 0 & 0
\end{array}\right] \\
& =\underbrace{\left[\begin{array}{llll}
G_{1} & G_{2} & 0 & 0
\end{array}\right]}_{G_{O}} \underbrace{\operatorname{diag}(Q, U, I, I)}_{\bar{T}}
\end{aligned}
$$

where $G_{O} \in \mathbb{R}^{\left(n_{\text {aw }}+n_{u}\right) \times\left(n+n_{u}+n_{z}+n_{w}\right)}$ and $\bar{T} \in$ $\mathbb{R}^{\left(n+n_{u}+n_{z}+n_{w}\right) \times\left(n+n_{u}+n_{z}+n_{w}\right)}$. Since $\bar{T}$ is invertible (indeed, $Q>0$ and $U>0$ by assumption), we can write

$$
W_{G}^{T} \Psi W_{G}=W_{G}^{T} \bar{T} \underbrace{\bar{T}^{-1} \Psi \bar{T}^{-1}}_{\bar{\Phi}} \underbrace{\bar{T} W_{G}}_{W_{G_{O}}}=W_{G_{O}}^{T} \bar{\Psi} W_{G_{O}}
$$

where $W_{G_{O}}$ spans the null space of $G_{O}$ and, according to the definitions $P=Q^{-1}$ and $U=W^{-1}$ (36) shown at the bottom of the page holds. Based on (19b), we can write explicitly the entries of $G_{O}$ as

$$
G_{O}=\left[\begin{array}{llll}
G_{1} & G_{2} & 0 & 0
\end{array}\right]=\left[\begin{array}{cccccc}
0 & 0 & I_{n_{\mathrm{aw}}} & 0 & 0 & 0 \\
0 & 0 & 0 & I_{n_{u}} & 0 & 0
\end{array}\right] .
$$

Hence, a matrix $\quad W_{G_{O}} \quad \in$ $\mathbb{R}^{\left(n_{p}+n_{c}+n_{\mathrm{aw}}+n_{u}+n_{z}+n_{w}\right) \times\left(n_{p}+n_{c}+n_{z}+n_{w}\right)}$ that spans the null space of $G_{O}$ is

$$
W_{G_{O}}:=\left[\begin{array}{cccccc}
I_{n_{p}} & 0 & 0 & 0 & 0 & 0 \\
0 & I_{n_{c}} & 0 & 0 & 0 & 0 \\
0 & 0 & 0 & 0 & I_{n_{z}} & 0 \\
0 & 0 & 0 & 0 & 0 & I_{n_{w}}
\end{array}\right]^{T}
$$

Using the partition of the matrix

$$
P=\left[\begin{array}{ll}
P_{11} & P_{12} \\
P_{12}^{T} & P_{22}
\end{array}\right]
$$

we can compute explicitly the inequality (31b) based on the definitions (36) and (37) and substituting (19a) and (19c) into the entries of $\bar{\Psi}$. After some computations it follows that $W_{G}^{T} \Psi W_{G}<0$ coincides with the inequality in (11b), as desired.
Conditions (11e) and (11f): Since $P=Q^{-1}$, and $S=P_{11}^{-1}$, then from the partitions of $P$ and $Q$ we have

$$
Q=\left[\begin{array}{cc}
R & N \\
N^{T} & M
\end{array}\right]>0 \text { and } Q^{-1}=P=\left[\begin{array}{cc}
S^{-1} & P_{12} \\
P_{12}^{T} & P_{22}
\end{array}\right]
$$

which can be rewritten as follows:

$$
\left[\begin{array}{cc}
R & N \\
N^{T} & M
\end{array}\right]>0 \quad\left[\begin{array}{cc}
R & N \\
N^{T} & M
\end{array}\right]^{-1}=\left[\begin{array}{cc}
S^{-1} & P_{12} \\
P_{12}^{T} & P_{22}
\end{array}\right] .
$$

By virtue of Lemma 6 expressions (38) are equivalent to

$$
\begin{aligned}
S^{-1}-R^{-1} & \geq 0 \\
\operatorname{rank}\left[S^{-1}-R^{-1}\right] & \leq n_{\mathrm{aw}} .
\end{aligned}
$$

Premultiplying and postmultiplying the matrices in (39b) by $S$ and $R$, respectively and performing a Cholesky factorization (see, e.g., [28, p. 195],) on (39a), we get Conditions (11e) and (11f), thus completing the proof of the necessity part of item 1). To prove the sufficiency in item 1), the aforementioned reasoning can be reversed. In particular, conditions (11e) and (11f) imply (39), which by Lemma 6 imply the existence of $M, N$ satisfying (38). Finally, (11a) and (11b) hold with $\gamma \leq \tilde{\gamma}$, hence, by Lemma 5, inequality (30) holds too. This, in turn, implies that (10) is solvable.

Finally, we prove item 2) of the theorem. Since (22) coincides with (10) with the selection for $Q$ (16)-(18), then provided the matrix $Q$ satisfies expression (38), the proof of the sufficiency of item 1) can be followed verbatim to show that (22) is solvable with (16)-(18). To show that the construction (16)-(18) for $Q$ satisfies (38), note that by the formulae for the inversion of block matrices [31, p. 23], the upper left block of $P$ needs to satisfy

$$
P_{11}=S^{-1}=R^{-1}+R^{-1} N\left(M-N^{T} R^{-1} N\right)^{-1} N^{T} R^{-1}
$$

which, when premultiplied and postmultiplied by $R$ and substituting the selection (17) for $M$, becomes

$$
R+N N^{T}=R S^{-1} R
$$

which, by (16), is always satisfied.

\section{CONCLUSION}

The problem of synthesizing fixed-order antiwindup compensators which meet an $\mathcal{L}_{2}$ performance bound has been addressed. The main results have demonstrated how a Lyapunov formulation of this problem can be expressed as a nonconvex optimization problem which closely resembles the LMI formulation of $\mathcal{H}_{\infty}$ controller synthesis. For certain antiwindup compensator state dimensions, the optimization problem is actually convex and hence can be solved using standard methods, which allow the construction of an optimal compensator that achieves a maximum performance level globally, via convex optimization.

$$
\bar{\Psi}=\left[\begin{array}{cccc}
A_{\circ}^{T} P+P A_{\circ} & P B_{q \circ}+C_{y \circ}^{T} W & P B_{w} & C_{z \circ}^{T} \\
B_{q \circ}^{T} P+W C_{y \circ} & -2 W+W D_{y q \circ}+D_{y q \circ}^{T} W & W D_{y w} & D_{z q \circ}^{T} \\
B_{w}^{T} P & D_{y w}^{T} W & -\gamma I & D_{z w}^{T} \\
C_{z \circ} & D_{z q \circ} & D_{z w} & -\gamma I
\end{array}\right] .
$$




\section{REFERENCES}

[1] P. Apkarian and P. Gahinet, "A convex characterization of gain-scheduled $\mathcal{H}_{\infty}$ controllers," IEEE Trans. Automat. Contr., vol. 40, pp. 853-864, May 1995.

[2] K. J. Åström and L. Rundqwist, "Integrator windup and how to avoid it," in Proc. Amer. Control Conf., vol. 2, Pittsburgh, PA, June 1989, pp. $1693-1698$.

[3] G. Becker, A. Packard, D. Philbrick, and G. Balas, "Control of parametrically-dependent linear systems: A single quadratic Lyapunov approach," in Proc. Amer. Control Conf., vol. 3, San Francisco, CA, June 1993, pp. 2795-2799.

[4] S. Boyd, L. El. Ghaoui, E. Feron, and V. Balakrishnan", Linear Matrix Inequalities in System and Control Theory. Philadelphia, PA: SIAM, 1994.

[5] P. J. Campo, M. Morari, and C. N. Nett, "Multivariable antiwindup and bumpless transfer: A general theory," in Proc. Amer. Control Conf., vol. 2, Pittsburgh, PA, June 1989, pp. 1706-1711.

[6] S. Crawshaw and G. Vinnicombe, "Anti-windup synthesis for guaranteed $\mathcal{L}_{2}$ performance," in Proc. Conf. Decision Control, vol. 2, Sidney, NSW, Australia, Dec. 2000, pp. 1063-1068.

[7] C. Edwards and I. Postlethwaite, "Anti-windup and bumpless transfer schemes," in Proc. Int. Conf. Control, vol. 1, Sept. 1996, pp. 394-399.

[8] P. Gahinet and P. Apkarian, "A linear matrix inequality approach to $\mathcal{H}_{\infty}$ control," Int. J. Robust Nonlinear Control, vol. 4, no. 4, pp. 421-448, July-Aug. 1994.

[9] P. Gahinet, A. Nemirovski, A. J. Laub, and M. Chilali, LMI Control Toolbox. Natick, MA: The MathWorks, Inc., 1995.

[10] E. G. Gilbert, I. Kolmanovsky, and K. T. Tan, "Discrete-time reference governors and the nonlinear control of systems with state and control constraints," Int. J. Robust Nonlinear Control, vol. 5, no. 5, pp. 487-504, Aug. 1995.

[11] R. Hanus, "Antiwindup and bumpless transfer: A survey," in Proc. 12th IMACS World Congr., vol. 2, Paris, France, July 1988, pp. 59-65.

[12] T. Iwasaki and R. E. Skelton, "All controllers for the general $\mathcal{H}_{\infty}$ control problem: LMI existence conditions and state space formulas," $\mathrm{Au}$ tomatica, vol. 30, no. 8, pp. 1307-1317, Aug. 1994.

[13] P. Kapasouris, M. Athans, and G. Stein, "Design of feedback control systems for stable plants with saturating actuators," in Proc. Conf. Decision Control, vol. 1, Austin, TX, Dec. 1988, pp. 469-479.

[14] H. K. Khalil, Nonlinear Systems, 2nd ed. Upper Saddle River, NJ: Prentice-Hall, 1996.

[15] M. V. Kothare, P. J. Campo, M. Morari, and C. N. Nett, "A unified framework for the study of antiwindup designs," Automatica, vol. 30, no. 12, pp. 1869-1883, Dec. 1994

[16] M. V. Kothare and M. Morari, "Multivariable antiwindup controller synthesis using multi-objective optimization," in Proc. Amer. Control Conf., vol. 5, Albuquerque, NM, June 1997, pp. 3093-3097.

[17] _ - "Stability analysis of antiwindup control schemes: A review and some generalizations," presented at the Euro. Control Conf., Brussels, Belgium, July 1997.

[18] J. C. Lozier, "A steady-state approach to the theory of saturable servo systems," IRE Trans. Automat. Contr., pp. 19-39, May 1956.

[19] V. R. Marcopoli and S. M. Phillips, "Analysis and synthesis tools for a class of actuator-limited multivariable control systems: A linear matrix inequality approach," Int. J. Robust Nonlinear Control, vol. 6, no. 9-10, pp. 1045-1063, 1996

[20] S. Miyamoto and G. Vinnicombe, "Robust control of plants with saturation nonlinearity based on coprime factor representations," in Proc. 35th Conf. Decision Control, vol. 4, Kobe, Japan, Dec. 1996, pp. $2838-2840$.

[21] E. F. Mulder and M. V. Kothare, "Synthesis of stabilizing antiwindup controllers using piecewise quadratic Lyapunov functions," in Proc. Amer. Control Conf., vol. 5, Chicago, IL, June 2000, pp. 3239-3243.

[22] E. F. Mulder, M. V. Kothare, and M. Morari, "Multivariable antiwindup controller synthesis using linear matrix inequalities," Automatica, vol. 37, no. 9, pp. 1407-1416, Sept. 2001.

[23] A. Packard, "Gain scheduling via linear fractional transformations," Syst. Control Lett., vol. 22, no. 2, pp. 79-92, Feb. 1994.

[24] Y. Peng, D. Vrančić, R. Hanus, and S. S. R. Weller, "Anti-windup designs for multivariable controllers," Automatica, vol. 34, no. 12, pp. $1559-1565$, Dec. 1998

[25] C. Scherer, "The Riccati inequality and state-space $\mathcal{H}_{\infty}$-optimal control," Ph.D. dissertation, Univ. Wurzburg, Wurzburg, Germany, 1990.
[26] G. Scorletti and L. El. Ghaoui, "Improved LMI conditions for gain scheduling and related control problems," Int. J. Robust Nonlinear Control, vol. 8, no. 10, pp. 845-877, Aug. 1998.

[27] J. S. Shamma, "Anti-windup via constrained regulation with observers," Syst. Control Lett., vol. 40, no. 4, pp. 1869-1883, July 2000

[28] G. Strang, Linear Algebra and Its Applications, 3rd ed. New York: Harcourt Brace, 1988.

[29] A. R. Teel and N. Kapoor, "The $\mathcal{L}_{2}$ antiwindup problem: Its definition and solution," presented at the Euro. Control Conf., 1997.

[30] P. F. Weston and I. Postlethwaite, "Analysis and design of linear conditioning schemes for systems with nonlinear actuators," Dept. of Engineering, Leicester Univ., Leicester, U.K., vol. 98-6, Internal Report, 1998.

[31] K. Zhou, J. C. Doyle, and K. Glover, Robust and Optimal Control. $\quad$ Upper Saddle River, NJ: Prentice-Hall, 1995.

[32] F. Wu, K. M. Grigoriadis, and A. Packard, "Anti-windup controller design using linear parameter-varying control methods," Int. J. Control, vol. 73, no. 12, pp. 1104-1114, Aug. 2000.

[33] L. Zaccarian and A. R. Teel, "A common framework for antiwindup, bumpless transfer and reliable designs," Automatica, vol. 38, no. 10, 2002

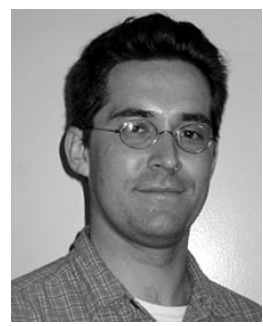

Gene Grimm (M'02) received the B.S. degree in engineering/physics from Westmont College, Santa Barbara, CA, in 1998 and the M.S. and Ph.D. degrees in electrical and computer engineering from the University of California, Santa Barbara, in 2002 and 2003 , respectively.

$\mathrm{He}$ recently joined the Space and Airborne Systems unit of Raytheon Company, El Segundo, CA.

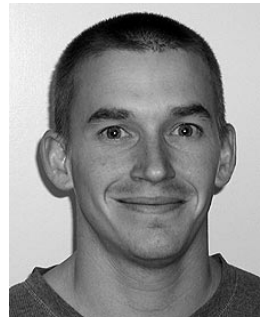

Jay Hatfield received the B.S. and M.S. degrees in electrical and computer engineering from the University of California, Santa Barbara, in 1999 and 2000, respectively.

Since 2000, he has been designing control systems for General Dynamics Land Systems at their facility in Goleta, CA.

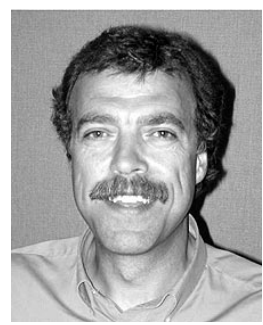

Ian Postlethwaite (M'79-SM'92-F'01) was born in Wigan, U.K., in 1953. He received the First Class B.Sc. (Eng.) degree from Imperial College, London University, London, U.K. in 1975 and the Ph.D. degree from Cambridge University, Cambridge, U.K., in 1978.

From 1978 to 1981, he was a Research Fellow at Cambridge University and spent six months at General Electric Company, Schenectady, NY. In 1981, he was appointed to a Lectureship in Engineering Science at Oxford University, Oxford, U.K., and in 1988 he became a Chair of Engineering at the University of Leicester, Leicester, U.K., where he is now Head of the Department. He has held short visiting positions at the University of California at Berkeley and at Santa Barbara, the Australian National University, Camberra, and the University of Auckland, Auckland, New Zealand. His research involves theoretical contributions to the field of robust multivariable control and the application of advanced control system design to engineering systems. He is a coauthor (with S. Skogestad) of Multivariable Feedback Control (New York: Wiley, 1996).

Dr. Postlethwaite is a Fellow of the IEE and the InstMC. In 1991, he received the IEE FC Williams premium; in 2001, he was awarded the Sir Harold Hartley Medal of the InstMC; and in 2002, he received a Best Paper Prize from the IFAC Journal of Control Engineering Practice. 


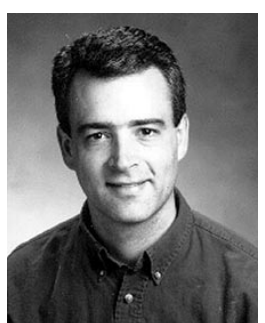

Andrew R. Teel (S'91-M'92-SM'99-F'02) received the A.B. degree in engineering sciences from Dartmouth College, Hanover, NH, in 1987 and the M.S. and Ph.D. degrees in electrical engineering from the University of California, Berkeley, in 1989 and 1992, respectively.

After receiving the Ph.D. degree, he was a Postdoctoral Fellow at the Ecole des Mines de Paris, Fontainebleau, France. In September 1992, he joined the faculty of the Electrical Engineering Department at the University of Minnesota, Minneapolis, where he was an Assistant Professor until September 1997. In 1997, he joined the Faculty of the Electrical and Computer Engineering Department at the University of California, Santa Barbara, where he is currently a Professor His research interests include nonlinear dynamical systems and control with application to aerospace and related systems.

Dr. Teel has received National Science Foundation Research Initiation and CAREER Awards, the 1998 IEEE Leon K. Kirchmayer Prize Paper Award, the 1998 George S. Axelby Outstanding Paper Award, and was the recipient of the first SIAM Control and Systems Theory Prize in 1998. He was also the recipien of the 1999 Donald P. Eckman Award and the 2001 O. Hugo Schuck Best Paper Award, both given by the American Automatic Control Council.

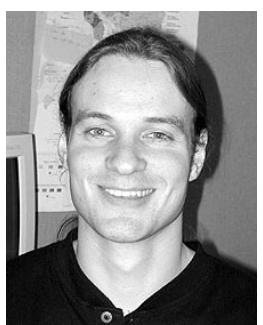

Matthew Turner (M'01) was born in Corby, U.K., in 1975. He received the B.Eng. degree in electrical and electronic engineering from the University of Surrey, Surrey, U.K., in 1996 and the Ph.D. degree in control engineering from the University of Leicester, Leicester, U.K., in 2000.

His main research interests are centered around robust control, flight control, and the control of systems containing isolated nonlinearities. He has designed and implemented robust controllers and antiwindup compensators on several industrial engineering systems and is currently involved with the European aerospace group, GARTEUR, in investigating the phenomenon of pilot-induced-oscillations.

Dr. Turner is a Member of the Institution of Electrical Engineers (IEE).

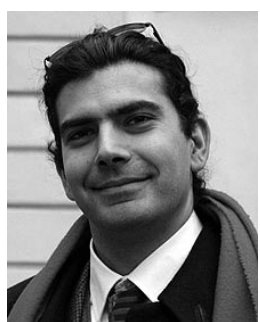

Luca Zaccarian graduated in electronic engineering from the University of Roma, Tor Vergata, Italy, in 1995 and he received the Ph.D. degree in computer science and control engineering from the same university in 2000.

During his Ph.D. studies, he spent approximately two years at the Center for Control Engineering and Computation of the University of California, Santa Barbara, where he conducted collaborative research projects on nonlinear control design. He is currently an Assistant Professor (Ricercatore) in compute science and control engineering at the University of Roma, Tor Vergata. His main research interests include analysis and design of nonlinear control systems, modeling and control of robots and mechanical systems, development of experimental robots, and real-time control systems.

Dr. Zaccarian was the recipient of the of the 2001 O. Hugo Schuck Best Paper Award given by the American Automatic Control Council. 